$\int_{1 / 2} 192050$

PPPL-2820

UC-426,427
PREPARED FOR THE U.S. DEPARTMENT OF ENERGY, UNDER CONTRACT DE-AC02-76-CHO-3073

PPPL-2820

\title{
ICRF STABILIZATION OF SAWTEETH ON TFTR
}

\section{BY}

C.K. PHILLIPS, J. HOSEA, E. MARMAR, M.W. PHILLIPS, ET. AL.

January. 1992

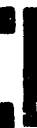

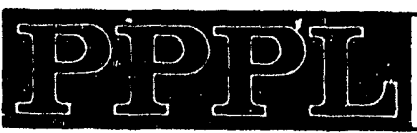

pDINCe:TON

plazma phygica

LABOAatofY

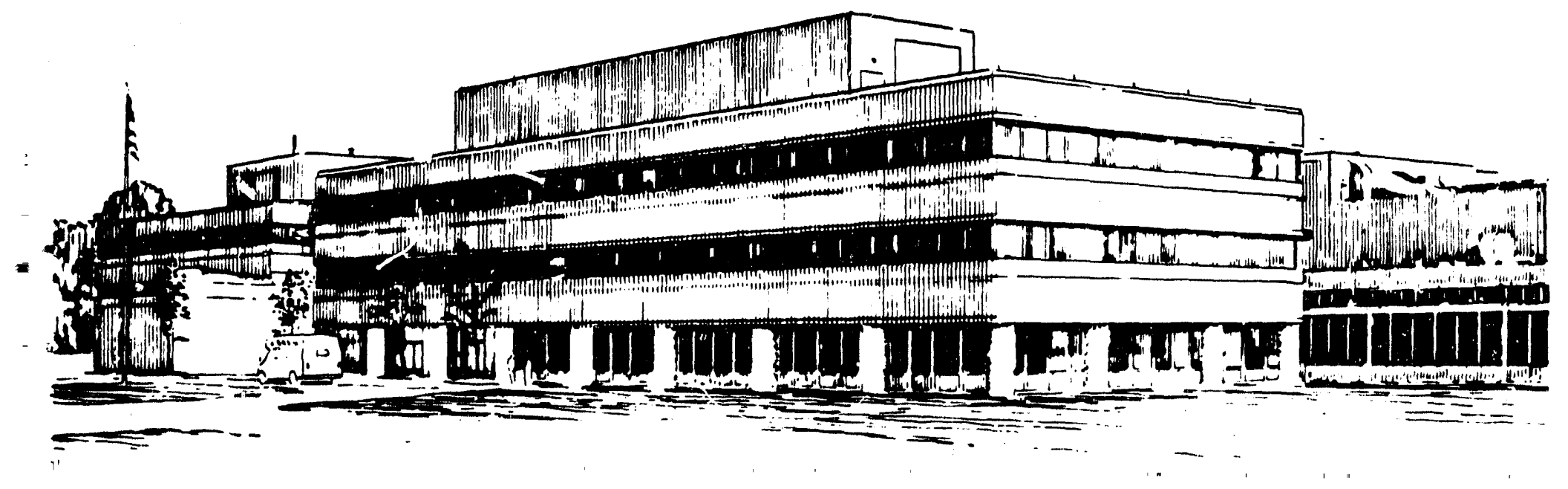




\section{NOTICE}

This report was prepared as an account of work sponsored by an agency of the United States Government. Neither the United States Government nor any agency, thereof, nor any of their employees makes any warranty, express or implied, or assumes any legal liability or responsibility for the accuracy, completeness, or usefulness of any information, apparatus, product, or process disclosed, or represents that its use would not infringe privately owned rights. Reference herein to any specific commercial produce, process, or service by trade name, trademark, manufacturer, or otherwise, does not necessarily constitute or imply its endorsement, recommendation, or favoring by the United States Government or any agency thereof. The views and opinions of authors expressed herein do not necessarily state or reflect those of the United States Government or any agency thereof.

\section{NOTICE}

This report has been reproduced directly from the best available copy.

Available to DOE and DOE contractors from the:

Office of Scientific and Technical Information P.O. Box 62

Oak Ridge, TN 37831 ;

Prices available from (615) 576-8401.

Available to the public from the:

National Technical Information Service

U.S. Department of Commerce 5285 Port Royal Road

Springfield, Virginia 22161

$703-487-4650$ 


\section{ICRF stabilization of sawteeth on TFTR}

C.K. Phillips, J. Hosea, E. Marmar ${ }^{a)}$, M.W. Phillips ${ }^{\text {b) }}$, J. Snipes ${ }^{\text {a)}}$, J. Stevens, J. Terry ${ }^{\text {a) }}$, J.R. Wilson, M. Bell, M. Bitter, R. Boivin ${ }^{\text {a) }}$, C. Bush ${ }^{\text {C) }}$, C.Z. Cheng, D. Darrow

E. Fredrickson,

R. Goldfinger ${ }^{c}$ ),

G. W. Hammett, K. Hill, D. Hoffman $^{\text {C) }}$, W. Houlberg ${ }^{C)}, H$. Hsuan, M. Hughes ${ }^{\text {b) }}$, D. Jassby, D. McCune, K. McGuire, Y. Nagayama d), D.K. Owens, H. Park, A. Ramsey, G. Schilling, J. Schivell, D.N. Smithe $\left.{ }^{\theta}\right)$, B. Stratton, E. Synakowski, G. Taylor, H. Towner, R. White, S. Zweben and the TFTR Group

Plasma Physics Laboratory, Princeton University, Princeton, New Jersey 08543

a) Massachusetts Institute of Technology, Cambridge, Massachusetts 02139

b) Grumman Corporation, 4 Independence Way, Princeton, New Jersey 08540

c) Oak Ridge National Laboratory, Oak Ridge, Tennessee 08731

d) University of Tokyo, Tokyo, Japan.

e) Mission Research Corporation, 8560 Cinderbed Rd., Suite 700, Newington, Virginia 22122

\section{ABSTRACT}

Results obtained from experiments utilizing high power ICRF (ion cyclotron range of frequency) heating to stabilize sawtooth oscillations on TFTR are reviewed. The key observations include existence of a minimum ICRF power required to achieve stabilization, a dependence of the stabilization threshold on the relative size of the ICRF power deposition profile to the $q=1$ volume, and a peaking of the equilibrium pressure and current profiles during sawtooth-free phases of the discharges. In addition, preliminary measurements of the poloidal magnetic field profile indicate that $q$ on axis decreases to a value of $0.55 \pm 0.15$ after a sawtooth-stabilized period of $\sim 0.5 \mathrm{sec}$ has transpired. The results are discussed in the 
context of theory, which suggests that the fast ions produced by the ICRF heating suppress sawteeth by stabilizing the $m=1$ MHD instabilities believed to be the trigger for the sawtooth oscillations. Though qualitative agreement is found between the observations and the theory, further re. finement of the theory coupled with more accurate measurements of experimental profiles will be required in order to complete quantitative comparisons. 


\section{INTRODUCTION}

The sawtooth instability, first observed on the PLT tokamak by von Goeler et al. in $1974^{1}$, adversely effects the performance of all tokamaks by periodically causing large scale transport in the plasma core. The repetitive flattening of the core region limits the peak temperatures, densities and fusion reactivity, $Q$, which can be achieved. During high power ICRF (ion cyclotron range of frequency) heating experiments on PLT, giant sawtooth oscillations led to a $30 \%$ modulation of the central electron and ion temperatures ${ }^{2}$, an observation which was later confirmed by experiments on JET ${ }^{3}$. Recently, the onset of sawtooth activity in TFTR at high plasma currents $\left(I_{p} \geq 1.8 \mathrm{MA}\right)$ has degraded the performance of high power neutral beam injection heated supershot plasmas ${ }^{4}$. Since the equivalent $Q_{D T}$ achieved in supershots on TFTR is maximized at high plasma currents ${ }^{4}$, an effective means of preventing sawteeth on TFTR in this regime is highly desirable. More generally, stabilization of sawtooth oscillations in the nearly ignited plasmas achievable in existing tokamaks and ultimately in tokamak fusion reactors may possibly lead to a more optimum performance of these devices.

Though numerous studies have been made to determine the exact nature and the dynamics of the sawtooth oscillation, controversy still remains in explaining the phenomenon ${ }^{5}$. One model that has gained wide acceptance is the Kadomstev model, in which the oscillations are initiated as the result of the growth of an unstable $m=n=1$ MHD mode when $q(r)$ drops below 1 in the plasma ${ }^{6}$. The application of RF heating power in various frequency regimes has been found to suppress sawtooth oscillations. Such stabilization was first observed on $T-10$ tokamak ${ }^{7}$ in experiments using ECRF (electron cyclotron range of frequency) heating, a result which has since been confirmed on other devices $8-12$. The stabilization was most effective when the ECRF heating was applied near the $q=1$ layer on the high field side of the magnetic axis. It is believed that modification of the local shear near the $q=1$ layer due to the ECRF heating suppresses the onset of the $m=n=1$ internal kink mode. Sawtooth stabilization (SST) has 
also been observed during lower hybrid wave current drive (LHCD) experiments on the PLT ${ }^{13}$, PETULA ${ }^{14}$ and JT-60 ${ }^{15}$ tokamaks. It has been proposed that the RF driven current broadened the current profile sufficiently so that $q(r)$ greater than 1 was maintained during the heating pulse, thereby preventing the occurrence of $m=n=1$ MHD modes. Finally, sawtooth stabilization using ICRF minority species heating was first observed on $\mathrm{JET}^{16}$ and subsequently verified on TFTR ${ }^{17}$. ICRF minority heating leads to the creation of an energetic, anisotropic ion population in the plasma. It has been suggested ${ }^{18-21}$ that the perpendicular pressure exerted by these energetic ions on the plasma suppresses the $m=1$ internal kink instabilities which are thought to trigger the sawtooth instability.

An objective of the TFTR research program is to explore the hot ion stabilization regime, particularly at high densities, to determine if it can be successfully utilized to maximize plasma performance during the $D-T$ operations phase on TFTR and ultimately in the future generations of tokamak devices. Experimental studies combined with theoretical analyses are being pursued in order to test the proposal that the stabilization is due to the presence of the hot ions as well as to develop a quantitative understanding of the phenomenon. In this paper, the key experimental observations on sawtooth stabilization in TFTR will be reviewed in Sec. II and discussed in the context of the existing theoretical model ${ }^{18-21}$ in Sec. III. A summary and concluding remarks are presented in Sec. IV.

\section{EXPERIMENTAL OBSERVATIONS}

Observations of the ICRF sawtooth stabilization phenomenon in TFTR to date are comparable to those reported by JET ${ }^{16}$. Stabilization has been observed in both deuterium (D) and helium-4 $\left({ }^{4} \mathrm{He}\right)$ majority plasmas with minority hydrogen $(H)$ heating, and in $D$ majority plasmas with minority helium-3 $\left({ }^{3} \mathrm{He}\right)$ heating in conjunction with deuterium neutral beam injection (NBI) heating. The stabilized phase has been maintained for as long as five or six energy confinement times, which are typically in the range of 200-250 msecs in the ICRF heating experiments: In some cases, the stabilization persists past the end of the ICRF heating pulse for time intervals on the order of the hot ion slowing down time. In other cases, giant sawteeth reoccur in the plasma prior to the end of the ICRF pulse. A par- 
ticular example of an ICRF sawtooth stabilized discharge in TFTR is shown in Fig. 1 . In this case, the stabilized phase terminated with a large sawtooth crash prior to the end of the RF pulse. For comparison purposes, the electron temperature profiles are given at the top and bottom of a sawtooth in the $\mathrm{OH}$ phase and at the end of stabilized phase. During the stabilized phase, the electron temperature evolved into a significantly hotter and more peaked profile than in the corresponding $\mathrm{OH}$ phase. The electron temperature inversion radius at the crash of the stabilized phase is considerably larger than in the $\mathrm{OH}$ crash, indicating that the current profile has also steepened, consistent with the evolution of the electron temperature profile. The same increase in the sawtooth inversion radius was observed over a wide range of plasma currents; as shown in Fig. 2. At each of the plasma currents examined, the largest final inversion radii, and by inference, the largest final $q=1$ volumes, were found in those discharges in which the stabilized phase persisted for the longest period of time.

Experimentally it has been found that a power threshold exists below which ICRF stabilization of the sawteeth is not observed. For $\mathrm{H}$ minority heating experiments, the threshold power in TFTR is about $2.2 \mathrm{MW}$ with $D$ majority plasmas and about $3.5 \mathrm{MW}$ with ${ }^{4} \mathrm{He}$ majority plasmas. Furthermore, it has been found that stabilization is achieved most readily with on-axis heating when the ICRF power deposition profile is wellpeaked within the $q=1$ volume. In particular, in $D-(H)$ heating experiments in which the ICRF power was held constant at about the threshold power, 2.2 MW, stabilization was observed only when the resonance layer was lo. cated at the magnetic axis, as shown in Fig. 3. The threshold power for stabilization was also found to be dependent on the plasma current, as indicated in Fig. 4. For on-axis heating experiments in ${ }^{4} \mathrm{He}-(\mathrm{H})$ plasmas, stabilization was achieved with approximately 3.5 MW of ICRF power at a plasma current of 1.4 MA, while no stabilization was observed up to the maximum available power of about $6 \mathrm{MW}$ at a lower plasma current, 1.2 MA.

ICRF heating in TFTR induces a small toroidal plasma rotation relative to the $\mathrm{OH}$ phase in the direction counter to the equilibrium plasma current. The rotation is measured with the horizontally viewing $x$-ray crystal detector. This effect has also been observed on JET ${ }^{22}$. The magnitude of the rotation cioes not appear to change when sawtooth stabilization occurs. An example showing the time dependence of the induced toroidal rotation 
speed is given in Fig. 5. In this example, sawtooth stabilization was achieved near the end of the ICRF pulse. The time evolution of the rotation is qualitatively the same in discharges in which no stabilization is found.

The stabilized phase terminates with a very rapid growth of the $m=1$ island, culminatir.g in an extremely rapid and spatially extensive sawtooth crash. In Figs. $6 a$ and $6 \mathrm{~b}$, a typical $\mathrm{OH}$ sawtooth crash is compared to the crash that occurs when ICRF stabilization is lost. The electron temperature contours determined from the electron cyclotron emission (ECE) measurements obtained with a grating polychrometer, are displayed for the same time interval and over the same spatial scale for the two events. The $\mathrm{OH}$ sawtooth is preceded by $m=1$ oscillations which are clearly visible for a few milliseconds prior to the crash phase. For this event, the crash is completed in about $100 \mu s e c s$. In the stabilized discharge, no $\mathrm{m}=1$ precursors are visible on the ECE contours. However, accorling to data from the 2D $x$-ray viewing system, an $m=1$ mode is detectable at about 10

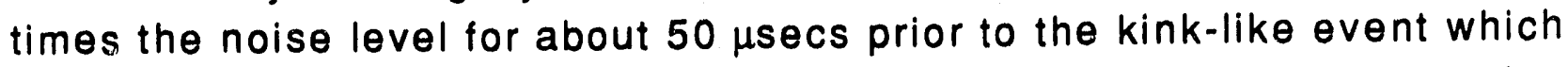
terminates the stabilized phase. Because of the small toroidal rotation speed in these discharges, as indicated in Fig. 5 above, the plasma does not rotate appreciably in the $50 \mu$ sec period in which the $m=1$ mode is present, thereby rendering it undetectable by the ECE system. The sawtooth crash in this case occurs in about $25 \mu$ secs. While $\mathrm{OH}$ and giant sawteeth can have crash phases which range from $40 \mu$ secs to a few milliseconds in time, the stabilized phase is always observed to terminate with a very rapid crash time, as shown in this example.

Preliminary measurements of the $q$ profile have been obtained at the end of a stabilized phase in collaboration with MIT using lithium (Li) pellet injection ${ }^{23}$. The technique involves measuring the spatial profile of the poloidal magnetic field $\left(B_{p}\right)$ by observing the orientation of the pellet ablatant emission cloud. Since the ions are confined by the equilibrium magnetic field, a cigar-shaped emission region forms around the pellet which is orientated along the direction of the total magnetic field. A q profile is inferred by combining the internal magnetic field profile with external magnetics data and iteratively solving the Grad-Shafranov equation to find a least-squares fit to the equilibrium. The equilibrium fits are obtained using the TokaMac code, written by $M$. Mauel ${ }^{24}$. In the TokaMac code, it is assumed that the equilibrium pressure profile, $p(\psi)$, 
and the flux function, $\mathrm{RB}_{T}(\psi)$, where $\psi$ is the radial coordinate of the flux surface, can be represented by low-order polynomials. Figure 7 shows the measured poloidal magnetic field profile for a $D-(H)$ plasma that was stabilized with 3 MW of ICRF power. The profile was obtained about 450 msecs after the beginning of the sawtooth-free phase of the discharge. Two Li pellets were injected into the plasma $5 \mathrm{msecs}$ apart in time; the profile was obtained from the emission cloud of the second pellet. In this case, the pellet penetrated beyond the magnetic axis of the discharge. The location where the measured poloidal magnetic field goes to zero, at about $R \sim 2.83 \pm 0.02 \mathrm{~m}$, agrees well with the location of the magnetic axis, at about $R \sim 2.82 \mathrm{~m}$, as deduced from a fit to the electron pressure profile. For the measured $B_{p}$ profile, the best fit obtained for the $q$ profile is shown in Fig. 8. The inferred value of $q$ on axis is $0.55 \pm 0.15$. This low value for $q(0)$ is consistent with the observed peaking of the electron temperature profiles, as shown above in Fig. 1, with the current profile peaking inferred on the basis of Fig. 2, as well as with TRANSP calculations of the current evolution in a similar discharge, as shown below in Fig. 9. Low values for $q(0)$ during ICRF stabilized discharges, as deduced from polarimetry measurements, have been reported as well by the JET team $^{25}$.

A number of inferences can be made on the basis of these observations. First of all, the occurrence of sawteeth can be avoided if ICRF heating is utilized to produce a population of sufficiently energetic ions which are spatially localized within the $q=1$ volume of the tokamak. The ease with which the stabilization can be achieved or maintained is dependent on the amount of ICRF power applied to the plasma, on the location of the ICRF resonance layer, and on the size of the $q=1$ volume. Secsnd, by preventing the occurrence of sawteeth, the electron temperature is allowed to evolve to a highly peaked profile, due to the centralized nature of the applied ICRF heating. Finally, during the sawtooth-free phase of the discharge, current evolution, which proceeds on the relatively long magnetic diffusion time scale, leads to a highly peaked equilibrium current profilo and a corresponding low value for $q$ on axis. In the next section, these trends will be discussed in the context of a theoretical explanation of the phenomenon. 


\section{COMPARISONS TO A THEORETICAL MODEL}

Though some of the original theoretical studies of the effects of an energetic trapped ion species on a tokamak plasma 26,27 were concerned with the onset of fishbone instabilities observed during NBI heating experiments in PDX ${ }^{28}$, a closer examination of the theory ${ }^{18-21}$ showed that the same formalism could be used to explain the sawtooth stabilization observed during ICRF heating experiments on JET ${ }^{16}$ and TFTR ${ }^{17}$. The effect of the energetic trapped ions on the MHD stability of the plasma are described using a variational formulation in which the plasma is considered to consist of a warm, background component governed by MHD theory plus a hot perturbation component treated with a gyrokinetic formalism ${ }^{18-21,26,27}$. In the ideal limit, the $m=1$ internal mode dispersion relation, modified by the presence of the hot ions, can be written as :

$$
\delta W_{c}+\delta W_{k}-\frac{i\left[\omega\left(\omega-\omega_{*_{i}}\right)\right]^{1 / 2}}{\omega_{A}}=0
$$

where $\delta W_{c}$ is the contribution from the bulk plasma, $\delta W_{k}$ is the kinetic contribution from the fast ions, $\omega_{A}$ is the shear Alfven frequency, $\omega_{*_{i}}$ is the thermal ion diamagnetic frequency, and $\omega$ is the mode frequency. The bulk term can be written in terms of the ideal growth rate as $-\gamma_{1} / \omega_{A}$. Assuming that the energetic trapped minority tail ions can be described by an equivalent Maxwellian distribution function and that the equilibrium can be modeled with circular flux surfaces in the low beta limit, then the dispersion relation (1) can be written in the form:

$$
\hat{\gamma}_{1}+i\left[\Omega\left(\Omega-\Omega *_{i}\right)\right]^{1 / 2}=\mathrm{A}\left(\begin{array}{c}
r_{1} \\
-1 \\
r_{h}
\end{array}\right)\left\langle\hat{\beta}_{p h}\right\rangle_{r_{1}} g(\Omega)
$$

where the constant $A$ and the function $g(\Omega)$ depends on the exact form of the hot particle distribution function, ${ }_{h}, r_{1}$ is the $q=1$ radius, $r_{h}$ is the effective gradient scale length for the hot particle perpendicular pressure, $p_{\perp h}$, within the $q=1$ volume, 


$$
r_{h}=\left[\frac{-1}{p_{\perp h}} \frac{\partial p_{\perp h}}{\partial r}\right]_{r_{1}}^{-1}
$$

$\left\langle\widehat{\beta}_{p h}\right\rangle_{r_{1}}$ is the normalized beta-poloidal for the hot ions averaged over the $q=1$ volume,

$$
\left\langle\hat{\beta}_{p h}\right\rangle_{r_{1}}=\varepsilon_{1} \frac{\omega_{A}}{\omega_{D}} \frac{2}{r_{1}^{2}} \int_{0}^{r_{1}} r d r \frac{p_{\perp h}}{B_{p}^{2} / 8 \pi},
$$

$\varepsilon_{1}$ is the inverse aspect ratio evaluated at the $q=1$ surface, $\Omega=\omega / \omega_{D}, \Omega_{*_{i}}$ $=\omega_{*_{i}} / \omega_{D}$, and $\omega_{D}$ is the bounce-averaged hot ion drift frequency, averaged over the hot particle distribution function. For deeply trapped ions, $\omega_{D}$ is given by:

$$
\omega_{D}=\left\langle\frac{E}{m r_{1} R \omega_{c}}\right\rangle_{r_{h}}
$$

where $E$ is the hot ion energy, $m$ is the hot ion mass, $R$ is the major radius, $\omega_{c}$ is the hot ion cyclotron frequency, and $\langle\ldots\rangle_{f_{h}}$ denotes a velocity space average over the hot particle distribution function.

The experimental observations described in the previous section are qualitatively consistent with certain features of the hot ion stabilization theory outlined above. By analyzing ${ }^{18-21}$ the modified dispersion relation for the $m=1$ modes given in Eq. (1), the energetic minority ion species were found to have a stabilizing influence only if the effective temperature, $T_{h}$, of the minority ions was much larger than that of the bulk ions, $T_{i}$, i.e.,

$$
\frac{T_{1}}{T_{h}} \sim O\left(\varepsilon^{2}\right)
$$


According to the simple Stix modol ${ }^{29}$, the minority ion temperature is determined by the volume-averaged ICRF power density applied to the plasma, $\left\langle p_{\mathrm{RF}}>\right.$ as well as by the density of the minority species, $n_{\min }$, and the hot ion slowing down time, $\tau_{\text {slow }}$ :

$$
T_{h} \sim \frac{\left\langle p_{\text {RF }}\right\rangle \tau_{\text {slow }}}{3 n_{\min }}
$$

From this expression, it is easy to see that for a given target plasma, that the minority ions will be heated to a sufficiently high temperature to achieve stabilization of the $m=1$ modes only if enough ICRF power is applied. Indeed, a power threshold for sawtooth stabilization has been observed in the experiments, as shown previously in Figs. 3 and 4 . The thermal ion temperature was typically between 3 and $6 \mathrm{keV}$ in the TFTR experiments, while the minority tail temperature was typically between 0.3 and $1 \mathrm{MeV}$.

A second prediction of the theoretical model is that stabilization is more effective if the hot ion pressure profile is well-peaked within the $q=1$ volume. This can be seen by noting that the kinetic contribution to the dispersion relation, $\delta W_{k}$, scales as $r_{1} / r_{h}$. In the TFTR experiments, the best results were obtained with on-axis heating, as indicated in Fig. 3. This dependence of $\delta W_{k}$ on $r_{1} / r_{h}$ is also consistent with the observation that stabilization is more difficult at lower plasma currents, as shown in Fig. 4. The $q=1$ radius in the $O H$ target plasma scales linearly with the plasma current. In Fig. 10, the relative locations of the $q=1$ radii for 1.2 and 1.4 MA are shown on a typical hot ion pressure profile that is calculated for the TFTR cases of Fig. 4. The kinetic stabilizing term is smaller for 1.2 MA, since $r_{1}$ is smaller and the effective hot ion pressure gradient, ${ }^{r} h$, within the $q=1$ volume is larger. Hence, the observation that stabilization was not achieved at 1.2 MA but was at $1.4 \mathrm{MA}$, as shown in Fig.4, is consistent with the ICRF power deposition profile extending beyond the $q=1$ radius at the smaller current. Though the JET experiments also found that the longest stablized periods were obtained with on-axis heating ${ }^{21}$, the dependence at low plasma currents was not seen since the ICRF power 
deposition profiles on JET are always well-contained within the $q=1$ volume 21 .

Another prediction that can be made on the basis of the modified dispersion relation is that stabilization should become more difficurt for a fixed ICRF power deposition profile as the $q=1$ surface expands as a result of magnetic diffusion during sawtooth-free operation ${ }^{21}$. In principle, stabilization is obtained when the growth rate, $\gamma_{1}$, of the $m=1$ mode due to the contributions of the bulk plasma is reduced to zero by the kinetic stabilizing influence of the hot ions, $\delta W_{k}$. According to the analytic estimates of Bussac et al. ${ }^{30}$, the ideal growth rate scales with $r_{1}$ as:

$$
\gamma_{1}-\varepsilon_{1}^{2}\left(\beta_{p}^{2}-\beta_{p c}^{2}\right)
$$

where $\beta_{p c}{ }^{2}$ depends on the particular equilibrium pressure and current profiles of the tokamak. The kinetic contribution, $\delta W_{k}$, scales with $r_{1}$ and with the total perpendicular energy content, $W_{\perp h}$, in the minority ions as:

$$
\delta W_{k}-\varepsilon_{1}\left\langle\hat{\beta}_{p h}\right\rangle_{r_{1}}-\frac{W_{\perp h}}{r_{1}}
$$

where the hot particle beta scales like $W_{\perp h} / V_{1}$, with $V_{1}$ denoting the $q=1$ volume. For a fixed power deposition profile in a quasistatic equilibrium, the minority energy content, $W_{\perp h}$, will remain roughly constant. However, as the $q=1$ radius expands, the energy needed to stabilize the $m=1$ oscillations will increase as $r_{1}{ }^{3}$, according to Eqs. (8) and (9). Hence, it is anticipated that the stabilizing influence of the hot ions will decrease in time as the current profile evolves and eventually sawteeth will reoccur in the discharge. Consistent with this thesis is the observation that giant sawteeth reoccur in some of the sawtooth-stabilized discharges on TFTR prior to the cessation of the ICRF heating pulse, an example of which is shown in Fig. 1. 
Quantitative comparisons between the theoretical predictions and the experimental observations are difficult due to simplifications in the models as well as uncertainties in the data. Theory predicts that a stable operating regime exists that depends on the hot ion beta. The stable regime is roughly triangular in shape when plotted in $\gamma_{1}-\left\langle\hat{\beta}_{\mathrm{ph}}\right\rangle$ space, as depicted in Fig. 11. For sufficiently high minority ion betas, stabilization of the ideal $m=1$ internal kink mode, and by inference, sawtooth oscillations, is achieved. However, if the minority beta reaches sufficiently high values, then instability returns in the guise of fishbone oscillations. In Fig. 12, data points from ICRF sawtooth stabilized discharges on TFTR are compared to a theoretical estimate of the stability triangle. The ideal kink growth rates for the data points were numerically determined using the ARES MHD stability code ${ }^{31}$. The equilibrium pressure and current profiles as well as the hot minority pressure profile used in the analysis were obtained directly from measurements or else deduced with SNAP ${ }^{32}$ or TRANSP ${ }^{33}$ analyses of the discharges. Depending on the assumptions made concerning the detailed velocity and configuration space dependence of the hot ion pressure profile as well as the equilibrium pressure and current profiles, factors of two changes in the actual positioning of the stability triangle are easily found. For this reason, the triangular boundary is shown cross-hatched in Fig. 12. Similarly, the hot ion betas shown in the diagram are also uncertain, because the actual hot ion pressure profile is not directly measured. Hence, though consistency is found between the experimental measurements and the theoretically determined stable operating regime, quantitative confirmation of the theory remains elusive.

A final piece of evidence in support of the proposed hot ion stabilization theory is found by reconsidering the ICRF heating experiments which were conducted on the PLT tokamak ${ }^{2}$. In these experiments, sawtooth stabilization was not observed, even for coupled ICRF power levels up to 4.3 MW, though in some experiments the sawtooth period was observed to lengthen as the ICRF power was increased ${ }^{34}$. The reason why stabilization was not achieved can perhaps be found by examining the ICRF power deposition profiles obtained in these experiments. In Fig. 13, the time evolution of the measured central electron and ion temperatures for a $D$ $\left({ }^{3} \mathrm{He}\right)$ heating experiment in PLT with $2.6 \mathrm{MW}$ are compared with a simula- 
tion obtained with the WHIST/RAZE predictive transport code ${ }^{35}$. The corresponding ICRF power deposition profiles to the ions and the electrons at the bottom of a sawtooth crash are displayed in Fig. 14. The power deposition profiles at other times are only slightly peaked relative to the profiles shown in Fig. 14. Excellent agreement is found between the predicted amount of ICRF power going to the electrons, $1.5 \mathrm{~W} / \mathrm{cm}^{3}$, and the measured amount ${ }^{2}, 1.3 \mathrm{~W} / \mathrm{cm}^{3}$, determined from the initial rate of rise of the central electron temperature immediately followiris a sawtooth event. In this experiment, the sawtooth period was about 10 msecs while the fast ion slowing down time was on the order of 100 msecs. Because of the frequent periodic flattening of the plasma core temperatures due to the sawtooth oscillations, the fast ion distribution function never had a sufficient amount of time to evolve to a centrally peaked profile with a high effective temperature. Hence, the lack of sawtooth stabilization in the PLT experiments can be attributed to a minority ion beta profile which remained flat within the $q=1$ volume (so that $r_{h}$ tended towards infinity) and which was not sufficiently hot to completely suppress $m=1$ internal kink modes.

\section{SUMMARY AND CONCLUDING REMARKS}

Sawtooth stabilization has been observed on TFTR with the application of high power ICRF heating in both gas-fueled and neutral beam fueled discharges. The observations are compatible with those reported by $J E T^{16,21}$. Stabilization has been seen in $D-(H), D-\left({ }^{3} \mathrm{He}\right)$, and ${ }^{4} \mathrm{He}-(H) \mathrm{mi}$ nority heating experiments. A threshold power for achieving stabilization has been noted that depends on the equilibrium plasma current, on the details of the ICRF power deposition profile, and on the majority ion species. ICRF-induced toroidal plasma rotation remains small and unaffected by the onset of sawtoath-free operation. During the stabilized phase, the equilibrium pressure profile becomes more peaked due to the central focalization of the ICRF heating. The inversion radius measured at the end of the stabilized phase is significantly larger than the inversion radius of the last sawtooth prior to the onset of stabilization. The increase in the inversion radius implies that current evolution during the sawtooth-free period results in a more peaked equilibrium current profile. Preliminary measurements of the equilibrium poloidal magnetic field profile using $\mathrm{Li}$ pellet injection indicate that the value of $q$ on axis is in the range of 0.4 - 
0.7 after a significantly long sawtooth-free period has transpired. These results are consistent with TRANSP simulations of the current evolution in an ICRF sawtooth stabilized discharge. A very rapid and spatially extensive sawtooth crash always occurs at the end of a stabilized phase of operation. Prior to the start of the crash phase, which typically lasts about $25 \mu$ secs, an $m=1$ distortion of the equilibrium geometry exists at a very low amplitude for a brief time period, on the order of $50 \mu$ secs.

The experimental observations qualitatively support the proposal ${ }^{18-}$ 21 that the stabilization of the $m=1$ internal kink modes, thought to trigger the sawtooth instability, can be attributed to the perpenticular pressure exerted by the hot trapped minority ions created with the ICRF heating. Various predictions of the theory are consistent with trends noted in the experiments. In particular, the existence of a power threshold is consistent with the theoretical requirement that the minority ion beta be sufficiently hot to stabilize the ideal $m=1$ modes. The dependence of the power threshold on plasma current, the loss of stabilization as the $q=1$ vislume increases, and the observation that stabilization is achieved most readily with on-axis heating are all consistent with the theoretical conclusion that the stabilizing influence of the hot ions scales with the ratio $r_{1} / r_{h}$, where $r_{1}$ is the $q=1$ radius and $r_{h}$ is the gradient scale length of the hot ion pressure profile within the $q=1$ volume. However, quantitative confirmation of the theory remains elusive, due to simplifications in the models as well as uncertainties in the experimental measurements. Much of the uncertainty can be attributed to the treatment of the hot ion pressure profile. The analytic dispersion relation used for the comparisons in this paper was derived using the assumption that the hot ion distribution function can be represented using an equivalent Maxwellian characterized by the effective temperature of the energetic tail ions. Improvements to the modeling are needed to include effects related to velocity space anisotropy as well as large banana width effects ${ }^{21}$. Furthermore, the analytic estimate of the kinetic contribution to the dispersion relation was also constructed assuming a low beta tokamak equilibrium with circular flux surfaces. More precise estimates of the stable operating regime will require the use of a numerical code, such as NOVA- $\mathrm{K}^{20}$, to address some of these issues. Finally, more accurate measurements of the equilibrium profiles and of the hot ion distribution function are also required in order to test the theory more rigorously. 
In conclusion, statilization of sawteeth using ICRF heating represents a promising method of enhancing the performance of tokamak plasmas, particularly in the reactor regime. Experiments are in progress on TFTR to determine if it is possible to use the method to extend the operation of NBI driven supershot plasmas to the high plasma current regime. In supershot plasmas on TFTR, the highest equivalent $Q_{D T}$ 's and highest $\beta_{\alpha}$ 's are

obtained at the highest plasma currents ${ }^{4}$. At plasma currents below 1.8 MA, sawtooth-free operation is achieved in the supershots with NBI heating alone. However, above $1.8 \mathrm{MA}$, the onset of sawteeth has effectively spoiled the performance of these plasmas ${ }^{4}$. Initial results from experiments in which supershot-like plasmas with high plasma current, 2.0 MA, and moderate $\mathrm{NBI}$ heating power, $11 \mathrm{MW}$, were subjected to an additional $3 \mathrm{MW}$ of ICRF heating are encouraging. In Fig. 15, the time evolutions of two companion shots, with and without the ICRF heating, are displayed. In the plasma heated with NBI only, a single large sawtooth event occurred midway through the heating pulse. The addition of the ICRF heating in the companion shot delayed the onset of the sawtooth untii the end of the ICRF heating pulse. With the upgrade of the ICRF heating system to $12.5 \mathrm{MW}$ in the spring of 1992, the experiments will be repeated with the full NBI heating power ( $33 \mathrm{MW})$ available on TFTR in order to determine the operating scenarios which will lead to the maximum fusion output and highest alpha particle beta when D-T operations are begun on TFTR in 1993.

\section{ACKNOWLEDGMENTS}

We wish to acknowledge the outstanding technical contributions of the TFTR and ICRF staff and the continued support of Drs. R. Davidson, R. Hawryluk, D. Meacie, and P. Rutherford for the ICRF project. We also wish to thank Drs. B. Coppi, F. Porcelli, and F. Romanelli for helping to elucidate many aspects of the theory discussed in this paper. This work was supported by U.S. D.O.E. Contract \#DE-AC02-76-CHO-3073. 
${ }^{1}$ S. von Goeler, W. Stodiek, and N. Sauthoff, Phys. Rev. Lett. 33,120 (1974).

${ }^{2}$ E. Mazzucato, R. Bell, M. Bitter, A. Cavallo, S. Cohen, P. Colestock, G. Greene, G. Hammett, E. Hinnov, J. Hosea, D. Hwang, R. Kaita, S. Lippmann, J. Lovberg, D. McNeill, D. Mueller, M. Ono, C.K. Phillips, G. Schilling, C. Skinner, S. Suckewer, and J.R. Wilson, in Plasma Physics and Controlled Nuclear Fusion Research 1984, Proceedings of the Tenth International Conference, London, 1984 (IAEA, Vienna, 1985), Vol. I, p. 433.

${ }^{3}$ R.D. Gill, D.V. Bartlett, G. Bracco, D.J. Campbell, S. Corti, A.E. Costley, A.W. Edwards, W. Engelhardt, H.-U. Fahrbach, D.J. Gambier, C.W. Gowers, R. Granetz, A. Hubbard, S.E. Kissel, N. Lopes-Cardozo, G. Magyar, J. O'Rourke, E. Oord, R. Prentice, S. Tsuji, B.J.D. Tubbing, A. Weller, J.A. Wesson, V. Zanza, and D. Zasche, in Proceedings of the Thirteenth European Conference on Controlled Fusion and Plasma Heating, Schliersee, West Germany, 1986, (European Physical Society, Petit-Lancy, Switzerland, 1986), Vol. 1, p. 21.

${ }^{4}$ R.J. Hawryluk, V. Arunasalam, C.W. Barnes, M. Beer, M. Bell, R. Bell, H. Biglari, M. Bitter, R. Boivin, N.L. Bretz, R. Budny, C.E. Bush, C.Z. Cheng, T.K. Chu, S.A. Cohen, S. Cowley, P.C. Efthimion, R.J. Fonck, E. Fredrickson, H.P. Furth, R.J. Goldston, G. Greene, B. Grek, L.R. Grisham, G. Hammett, W. Heidbrink, K.W. Hill, J. Hosea, R.A. Hulse, H. Hsuan, A. Janos, D. Jassby, F.C. Jobes, D.W. Johnson, L.C. Johnson, J. Kessner, C.K. Phillips, S.J. Kilpatrick, H. Kugel, P.H. Lamarche, B. LeBlanc, D.M. Manos, D.K. Mansfield, E.S. Marmar, E. Mazzucato, M.P. McCarthy, M. Mauel, D.C. McCune, K.M. McGuire, D.M. Meade, S.S. Medley, D.R. Mikkelsen, D. Monticello, R. Motley, D. Mueller, Y. Nagayama, G.A. Navratil, R. Nazikian, D.K. Owens, H. Park, W. Park, S. Paul, F. Perkins, S. Pitcher, A.T. Ramsey, M.H. Redi, G. Rewoldt, D. Roberts, A.L. Roquemore, P.H. Rutherford, S. Sabbagh, G. Schilling, J. Schivell, G.L. Schmidt, S.D. Scott, J. Snipes, J. Stevens, J.D. Strachan, B.C. Stratton, W. Stodiek, E. Synakowski, Y. Takase, W. Tang, G. Taylor, J. Terry, J.R. Timberlake, H.H. Towner, M. Ulrickson, S. von Goeler, R. Wieland, M. Williams, J.R. Wilson, K.L. Wong, M. Yamada, S. Yoshikawa, K.M. Young, M.C. Zarnstorff, and S.J. Zweben, to be published in Plasma Phys. Controlled Fusion.

${ }^{5}$ B.N. Kuvshinov and P.V. Savrukhin, Sov. J. Plasma Phys. 16, 353 (1990).

${ }^{6}$ B.B. Kadomstev, Fiz. Plazmy 1, 710 (1975). 
${ }^{7}$ H.W. Prekaar, R.M.J. Sillen et al., Proc. of Third International Workshop on ECE (Madison, WI, 1982) paper I.R. 82/056.

${ }^{8}$ G.A. Bobrovshii et al., Sov. J. Plasma Phys. 13, 665 (1987).

${ }^{9}$ TFR Group, Nucl. Fusion 28,1995 (1988).

${ }^{10}$ R.T. Snider et al., Phys. Fluids B1, 404 (1989).

${ }^{11}$ Y. Terumichi, H. Tanaka, K. Ogura, S. Ide, M. Iida, T. Itoh, M. Iwamasa, K. Hanada, H. Sakakibara, T. Minami, M. Yoshida, T. Maekawa, and S. Tanaka, in Radio-Frequency Power in Plasmas, AlP Conference Proceedings 190, Proc. of the Eighth Topical Conference, Irvine, CA 1989, p. 88.

${ }^{12}$ K. Hanada, H. Tanaka, M. lida, S. Ide, T. Minami, M. Nakamura, T. Maekawa, Y. Termuchi, S. Tanaka, M. Yamada, J. Manickam, and R. White, Phys. Rev. Lett. 66,1974 (1991).

${ }^{13}$ T.K. Chu, R. Bell, S. Bernabei, A. Cavallo, W. Hooke, J. Hosea, F. Jobes, E. Meservey, R. Motley, J. Stevens, S. von Goeler, and S. Guharay, in Radiofrequency Plasma Heating, AIP Conference Proceedings 129, Proc. Sixth Topical Conference, Callaway Gardens, GA 1985, p. 131.

${ }^{14}$ C. Gormezano, P. Blanc, H. Bottollier, P. Briand, G. Brifford, P. Chabert, M. Clement, A. Girard, W. Hess, G.T. Hoang, G. Ichtchenko, G. Melin, F. Parlange, J.C. Vallet, and D. Van Houtte, Ibid, p. 111.

15 JT-60 Team, Plasma Phys. Controlled Fusion 31, 1597 (1989).

${ }^{16}$ D.J. Campbell, D.F.H. Start, J.A. Wesson, D.V. Bartlett, V.P. Bhatnagar, M. Bures, J.G. Cordey, G.A. Cottrell, P.A. Dupperex, A.W. Edwards, C.D. Challis, C. Gormezano, C.W. Gowers, F.S. Granetz, J.H. Hamnen, T. Hellsten, J. Jacquinot, E. Lazzaro, P.J. Lomas, N. Lopes Cardozo, P. Mantica, J.A. Snipes, D. Stork, P.E. Stott. P.R. Thomas, E. Thompson, K. Thomsen, and G. Tonetti, Phys. Rev. Lett. 60, 2148 (1988).

17 J.C. Hosea, C.K. Phillips, J.E. Stevens, J.R. Wilson, M. Bell, R. Boivin, A. Cavallo, P. Colestock, E. Fredrickson, G. Hammett, D. Hoffman, H. Hsuan, A. Janos, D. Jassby, F. Jobes, K. McGuire, D. Mueller, Y. Nagayama, K. Owens, H. Park, G. Schmidt, B. Stratton, G. Taylor, K.L. Wong, S. Zweben, and the TFTR Group, to be published in Proceedings of the Joint VarennaLausanne Int. Workshop on Theory of Fusion Plasmas, Varenna, Italy 1990.

${ }^{18}$ R.B. White, M.N. Bussac, and F. Romanelli, Phys. Rev. Lett. 62, 539 (1989). 
${ }^{19}$ B. Coppi, P. Detragiache, S. Migliuolo, F. Pegoraro, and F. Porcelli, Phys. Rev. Lett. 63, 2733 (1989).

${ }^{20}$ C.Z. Cheng, Phys. Fluids B 2, 1427 (1990).

${ }^{21} \mathrm{~F}$. Porcelli, to be published in Plasma Phys. Controlled Fusion; also F. Porcelli, D.J. Campbell, W.D. Diachenko, L.-G. Eriksson, J.Jacquinot, L.S. Levin, D.F.H. Start, A. Taroni, in Proceedings of the Seventeenth European Conference on Controlled Fusion and Plasma Heating, Amsterdam, The Netherlands, 1990, (European Physical Society, 1990), Vol. 14B, Part I, p. 327.

22 F. Bombarda, private communication.

${ }^{23}$ E.S. Marmar and J.L. Terry, Rev. Sci. Inst. 61, 3081 (1990).

$24 \mathrm{M}$. Mauel, private communication.

25 D.J. Campbell, J.G. Cordey, A.W. Edwards, R.D. Gill, E. Lazzaro, G. Magyar, A.L. McCarthy, J. O'Rourke, F. Pegoraro, F. Porcelli, P. Smeulders, D.F.H. Start, P. Stubberfield, J.A. Wesson. E. Westerhof, and D. Zasche, in Plasma Physics and Controlled Nuclear Fusion Research 1988, (IAEA, Vienna, 1989), Vol. 1, p. 377.

${ }^{26}$ L. Chen, R.B. White, and M.N. Rosenbluth, Phys. Rev. Lett. 52, 1122 (1984).

27 B. Coppi and F. Porcelli, Phys. Rev. Lett. 57, 2272 (1986).

28 K. McGuire et al., Phys. Rev. Lett 50, 891 (1983).

${ }^{29}$ T.H. Stix, Nucl. Fusion 15, 737 (1975).

${ }^{30}$ M.N. Bussac, R. Pellat, D. Edery, and J.L. Soule, Phys. Rev. Lett 35, 1638 (1975).

${ }^{31} M . W$. Phillips and M. Hughes, private communication.

${ }^{32}$ D.N. Smithe, C.K. Phillips, G.W. Hammett, and P.L. Colestock, in Radiofrequency Power in Plasmas, AIP Conference Proceedings 190, Proc. Eighth Topical Conference, Irvine, CA 1989, p. 338.

33

G.W. Hammett, P.L. Colestock, R.S. Granetz, P. Kupschus, D.C. McCune, C.K. Phillips, G.L. Schmidt, D.N. Smithe, and members of the JET/USDOE Pellet Collaboration, in Proceedings of the Sixteenth European Conference on Controlled Fusion and Plasma Heating, Vienna, Italy, 1989, (European Physical Society, 1989), p. 13.1. 
34 J.R. Wilson and the PLT Group, in Radiofrequency Plasma Heating, AIP Conference Proceedings 129, Proc. Sixth Topical Conference, Callaway Gardens, GA 1985, p. 28.

${ }^{35}$ D.G. Swanson, S. CHO, C.K. Phillips, D.Q. Hwang, W. Houlberg, and L. Hively, in Plasma Physics and Controlled Nuclear Fusion Research 1986, (IAEA, Vienna, 1989), Vol. 1, p. 653. 


\section{FIGURE CAPTIONS}

Fig. 1 The time evolution of the central electron temperature in an ICRF sawtooth stabilized discharge in TFTR is shown in (a). The radial profiles at the top and bottom of a sawtooth event are shown in (b) for the $\mathrm{OH}$ phase and in (c) at the end of the stabilized phase.

Fig. 2 The increase of the sawtooth inversion radius measured at the end of the stabilized phase relative to the size in the OH phase in shown for a number of discharges with different values of the equilibrium plasma current.

Fig. 3 The longest sawiooth period observed in $D-(H)$ heating experiments is plotted as a function of the location of the ICRF resonance layer. The ICRF power was approximately equal to the experimentally determined threshold level for achieving sawtooth stabilization. Stabilization is observed only with on-axis heating in these experiments.

Fig. 4 The longest sawtooth period obtained as a function of the applied ICRF heating power in ${ }^{4} \mathrm{He-}(\mathrm{H})$ heating experiments is shown for two different values of the equilibrium plasma current. The threshold power for stabilizing is higher than the observed threshold in $D-(H)$ heating experiments and is dependent on the value of the equilibrium plasma currént.

Fig. 5 The time evolution of the ICRF-induced toroidal rotation velocity is given for a discharge which entered the stabilized regime shortly before the termination of the ICRF heating pulse. The rotation speed, measured relative to the $\mathrm{OH}$ phase, is in the direction counter to the equilibrium current and remains small throughout the discharge.

Fig. 6 The ECE contours for an $\mathrm{OH}$ sawtooth event in (a) are compared to the crash at the end of a sawtooth-stabilized phase in (b).

Fig. 7 The poloidal magnetic field profile measured with $\mathrm{Li}$ pellet injection in a $D-(H)$ discharge stabilized with $3 \mathrm{MW}$ of ICRF heating is displayed. In this example, the pellet penetrated beyond the magnetic axis of the discharge. 
Fig. 8 The q profile corresponding to the poloidal magnetic field proflie given in Fig. 7 is shown. The value of $q(0)$ is in the range of $0.4-0.7$, with a best fir to the equilibrium obtained with $q(0) \sim 0.5$.

Fig. 9 TRANSP calculations of the current evolution of a sawtooth-stabilized discharge, shown in (a), are consistent with $q(0)$ dropping wellbelow 1 during sawtooth-free periods of operation.

Fig. 10 The locations of the $q=1$ radius in $\mathrm{OH}$ plasmas are shown relative to a typical perpendicular pressure profile of the energetic trapped ions created with ICRF heating in TFTR.

Fig. 11 The theoretically predicted sawtooth-stabilized operating regime is drawn in $\gamma_{1}-\beta_{\mathrm{ph}}$ space.

Fig. 12 The locations of ICRF-stabilized discharges on TFTR are plotted on the theoretical stability diagram. The direction of the arrow indicates the timie trajectory of a particular discharge through the stability diagram, as determined with TRANSP calculations of the hot lon pressure profile.

Fig. 13 Experimental measurements of the time evolution of the central electron and ion temperatures in a PLT heating experiment ${ }^{2}$ are compared to a WHIST/RAZE simulation of the experiment.

Fig. 14 The calculated ICRF power deposition profiles to the ions and electrons, are displayed at the bottom of a sawtooth crash for the PLT experiment shown in Fig. 13. The calculated rate of electron heating is in excellent agreement with the experimentally measured value ${ }^{2}$.

Fig. 15 The time evolution of the central electron temperature in a supershot-like plasma created with $11 \mathrm{MW}$ of NBI heating is compared to an identical discharge in which an additional $3 \mathrm{MW}$ of ICRF heating was applied. The effect of the ICRF heating was to delay the onset of the sawtooth crash until the end of the ICRF heating pulse. 


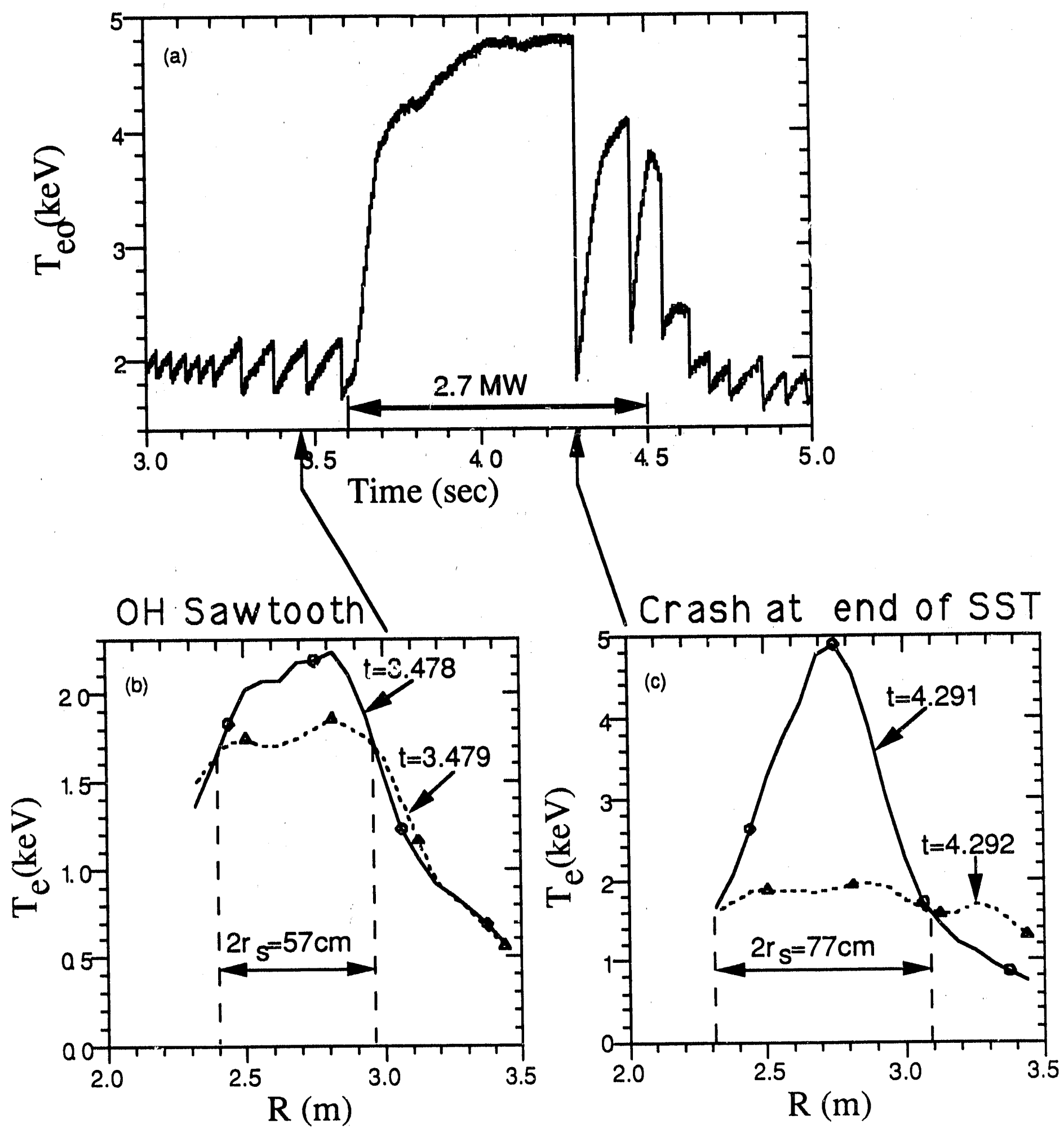

Fig. 1 


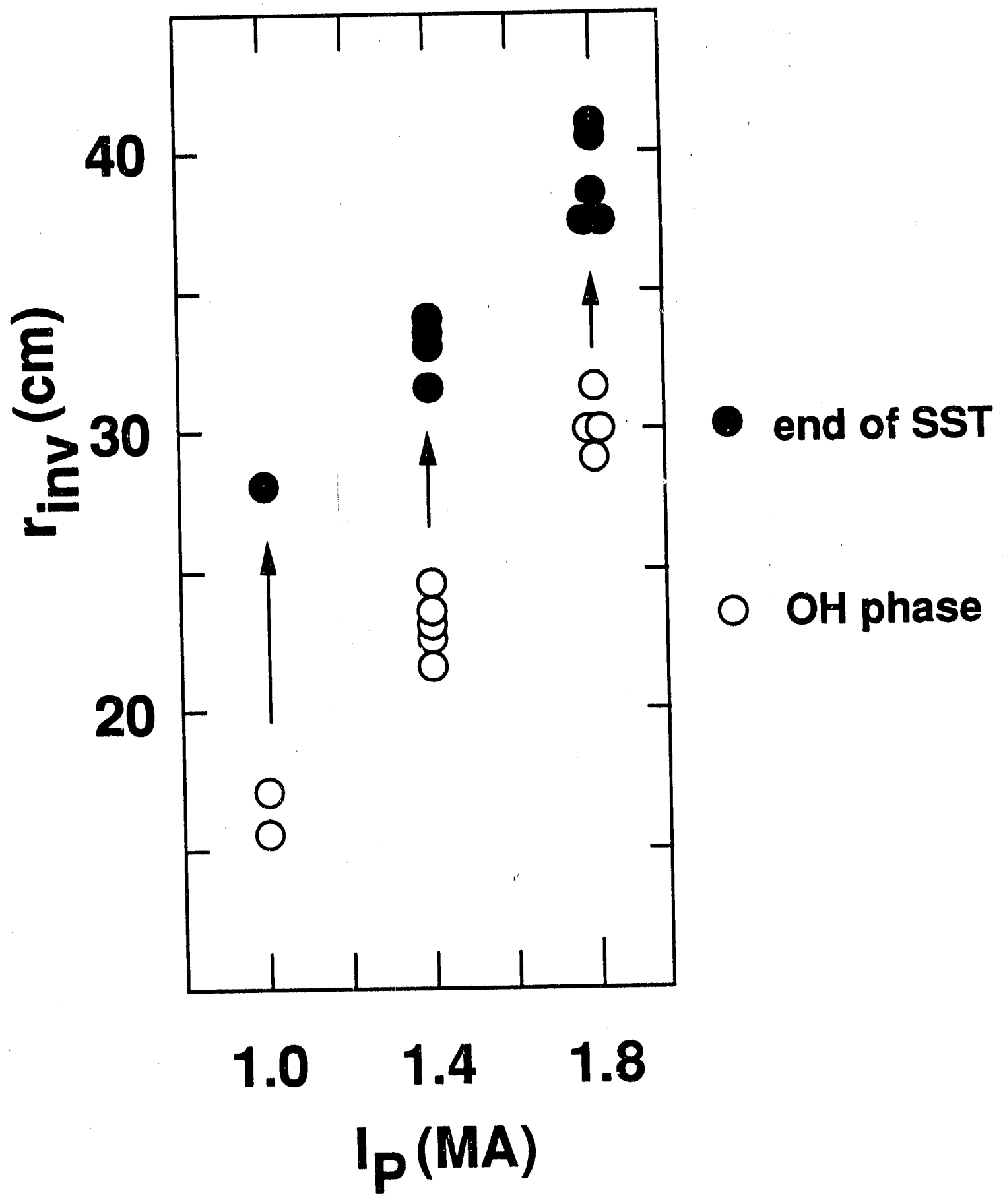

Fig. 2 


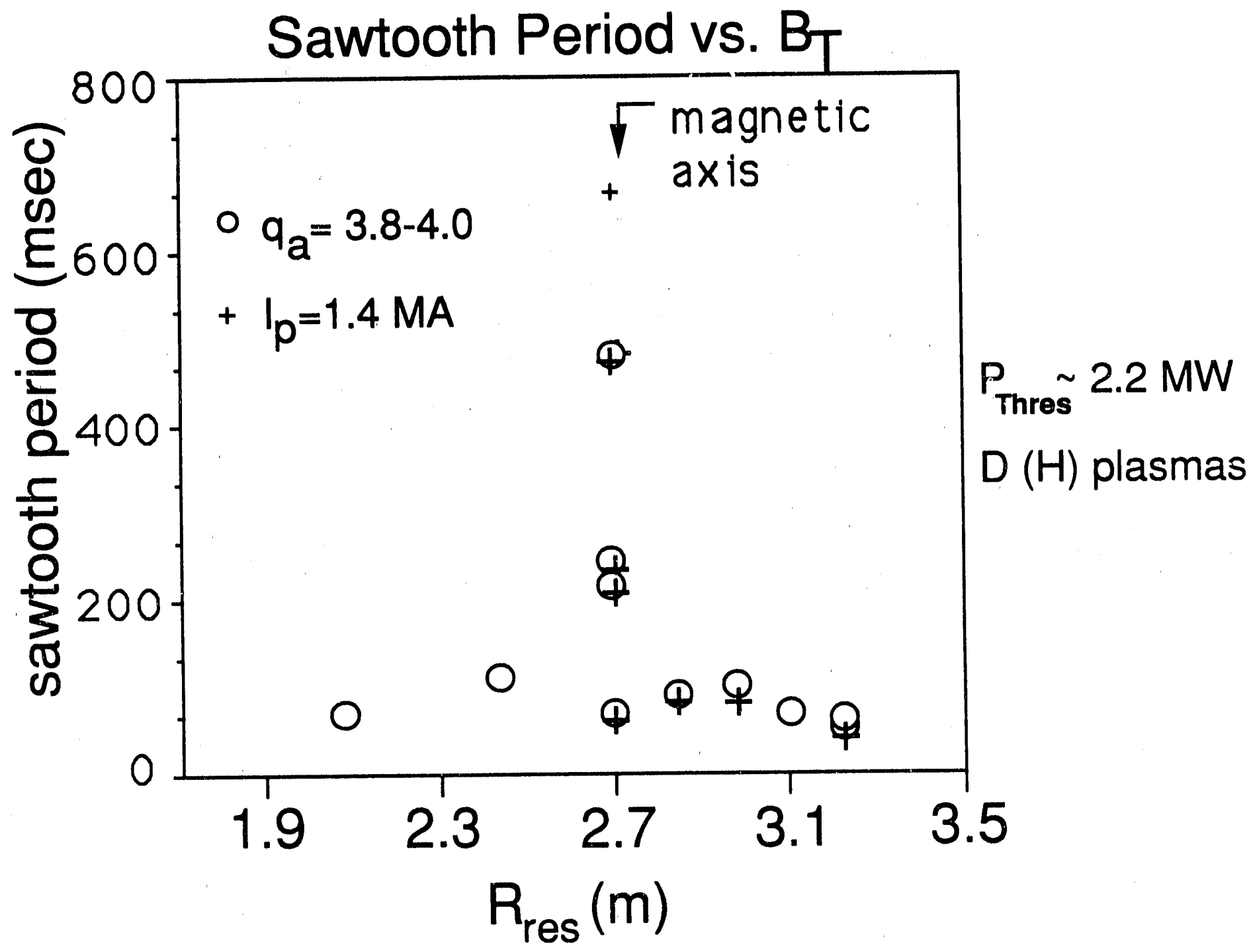

Fig. 3 


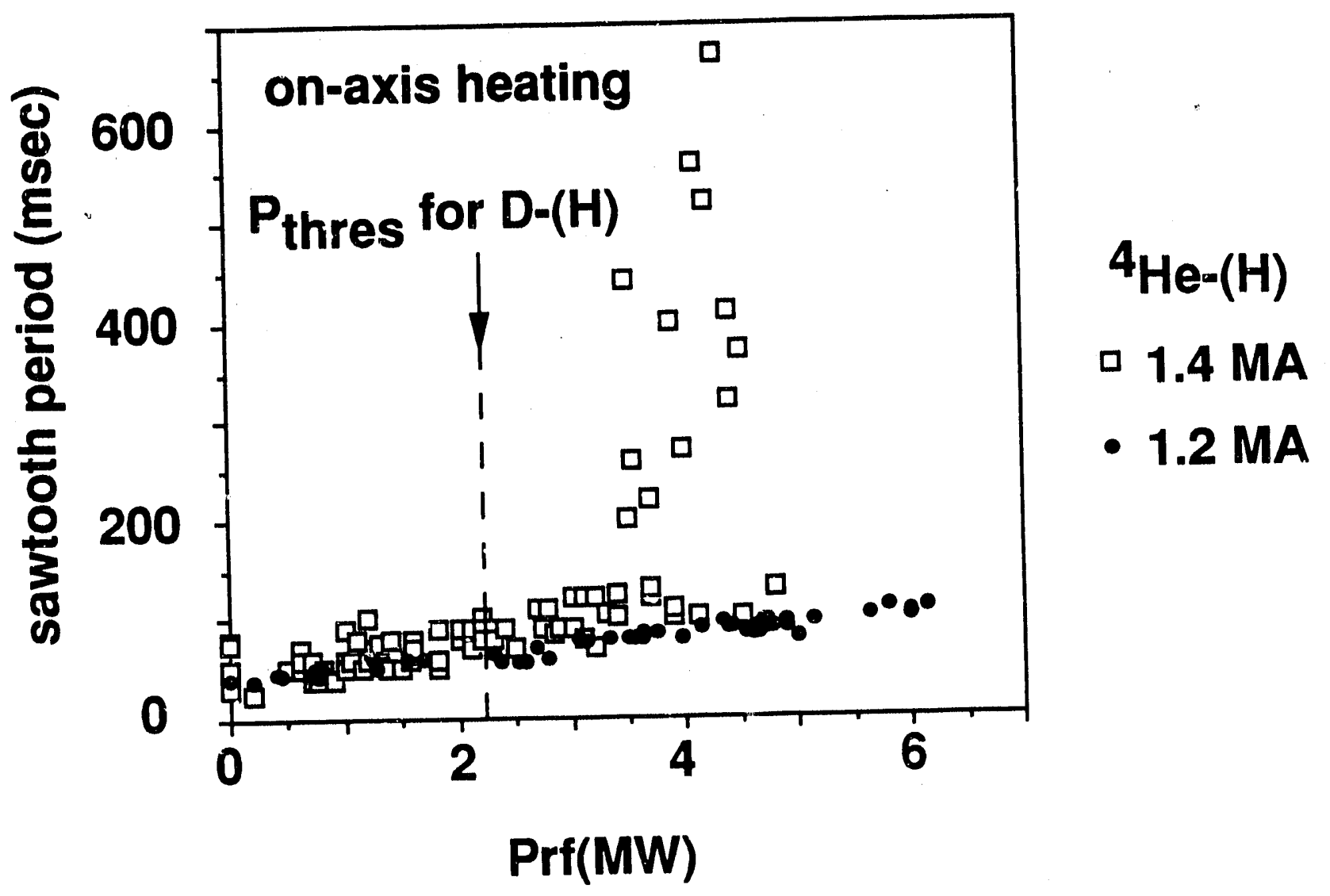

Fig. 4 


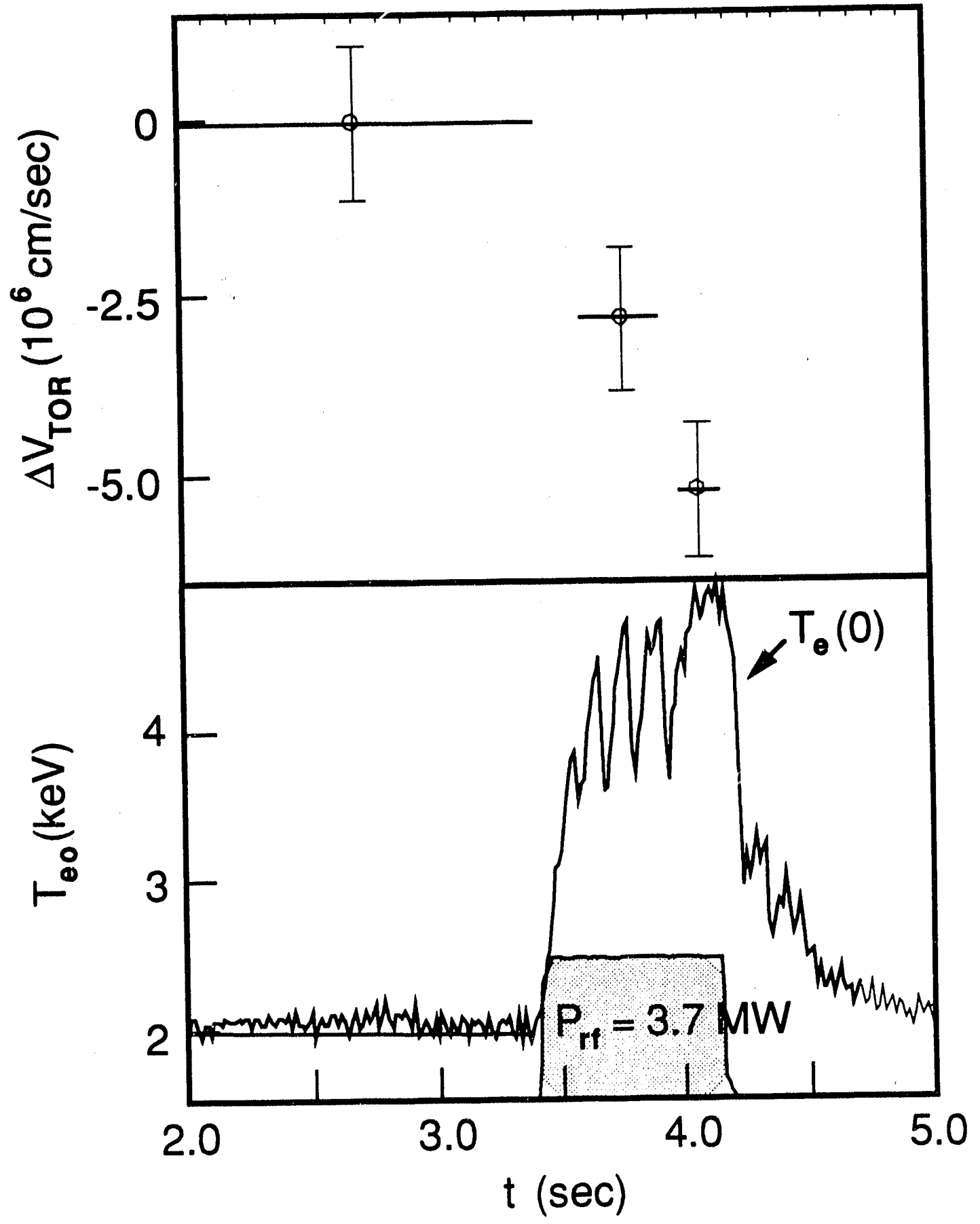

Fig. 5 
ECE Temperature Contours for an $\mathrm{OH}$ Sawtooth (shot 46191)

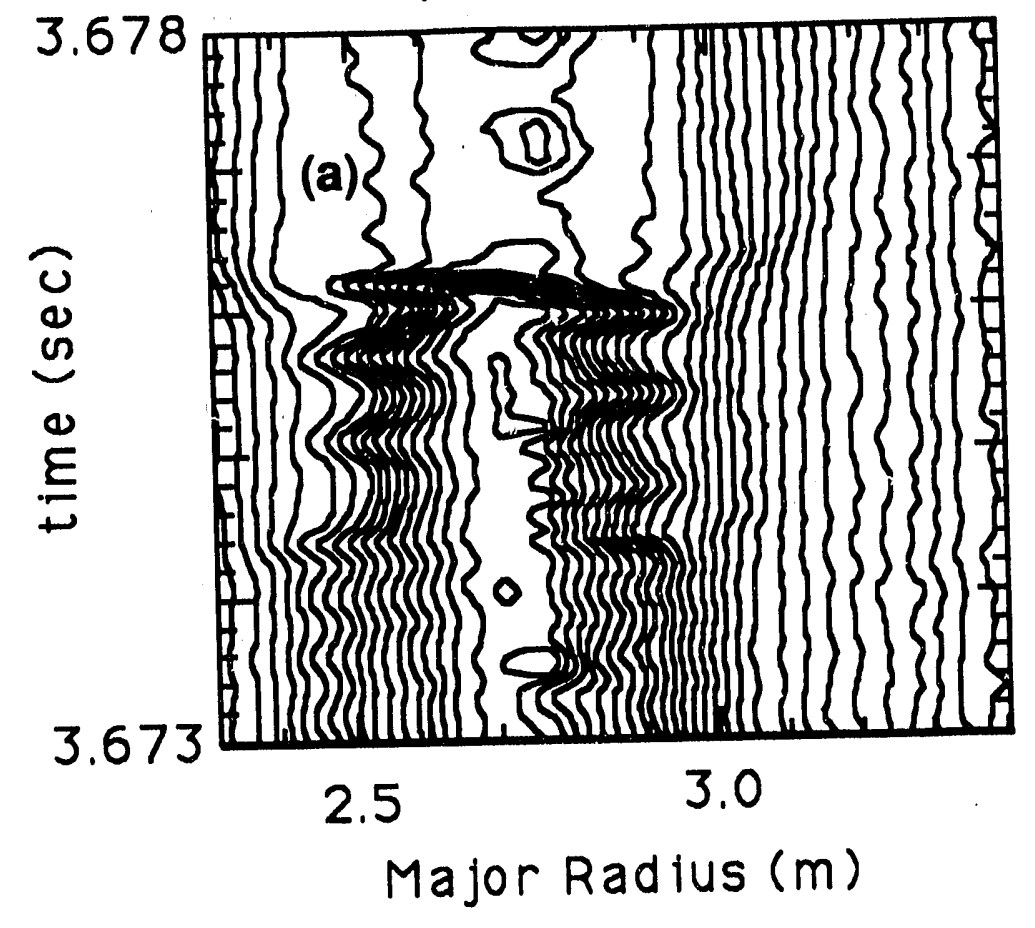

ECE Temperature Contours for CRASH of SST Phase (shot 46207)

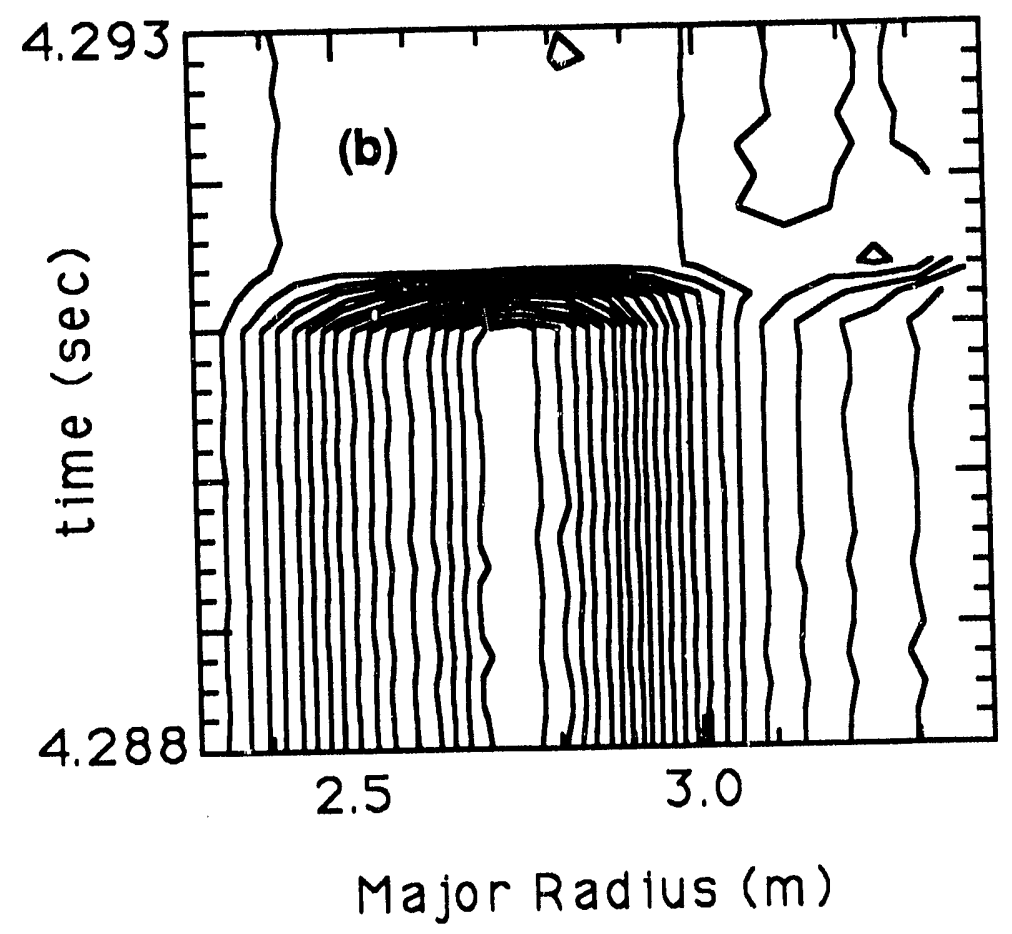

Fig. 6 


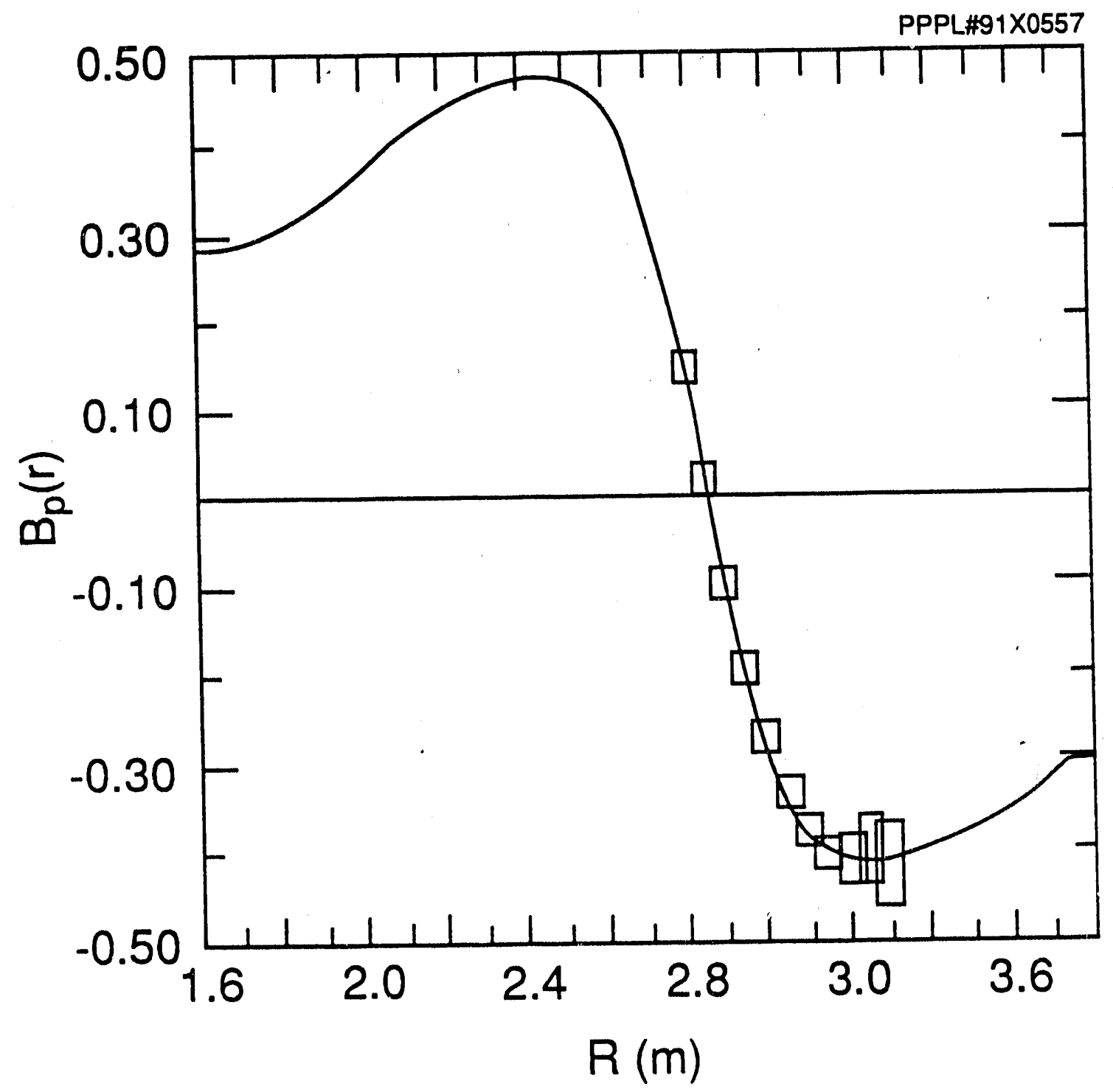

Fig. 7 


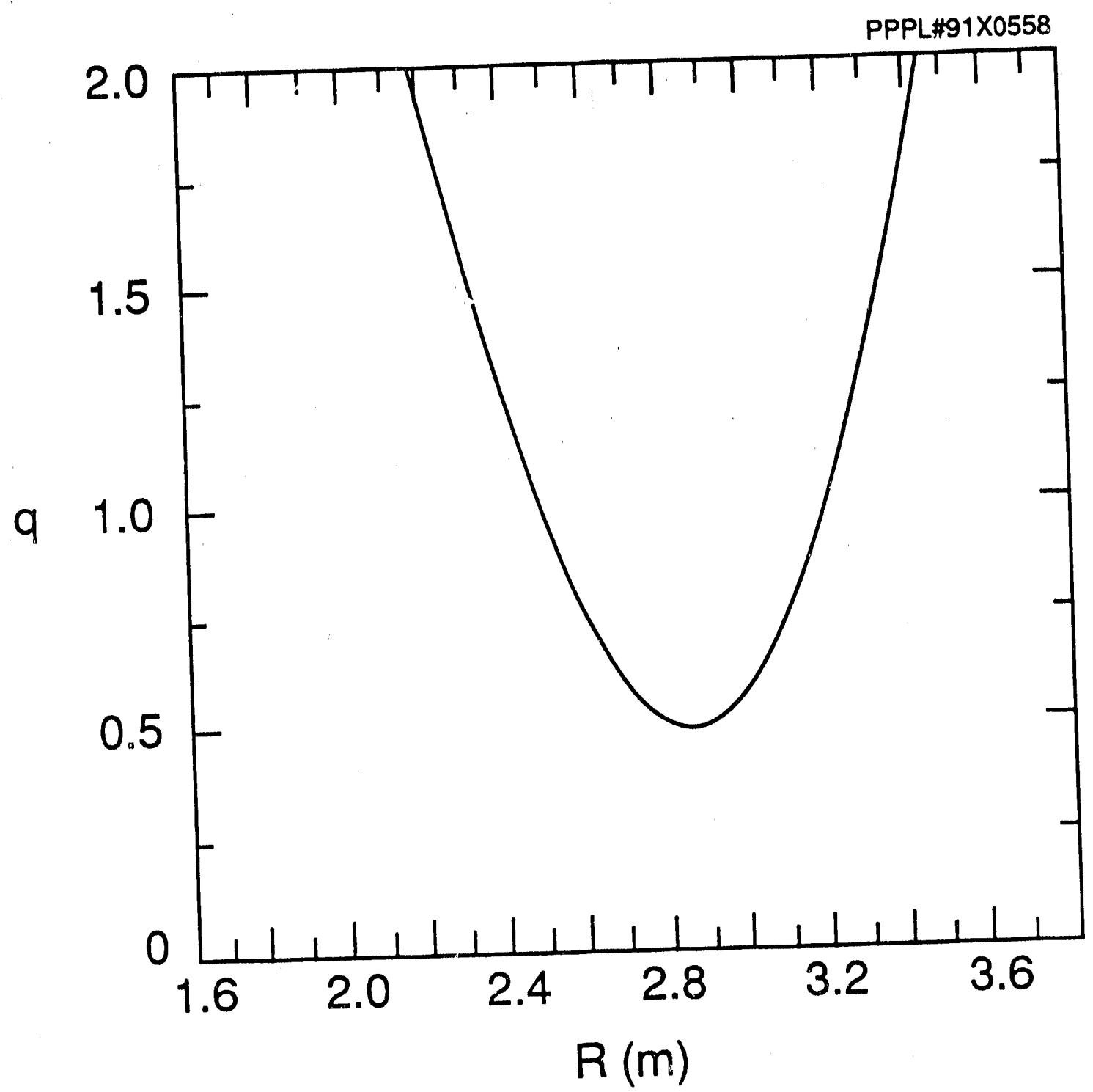

Fig. 8 
Central Electron Temperature vs. Time

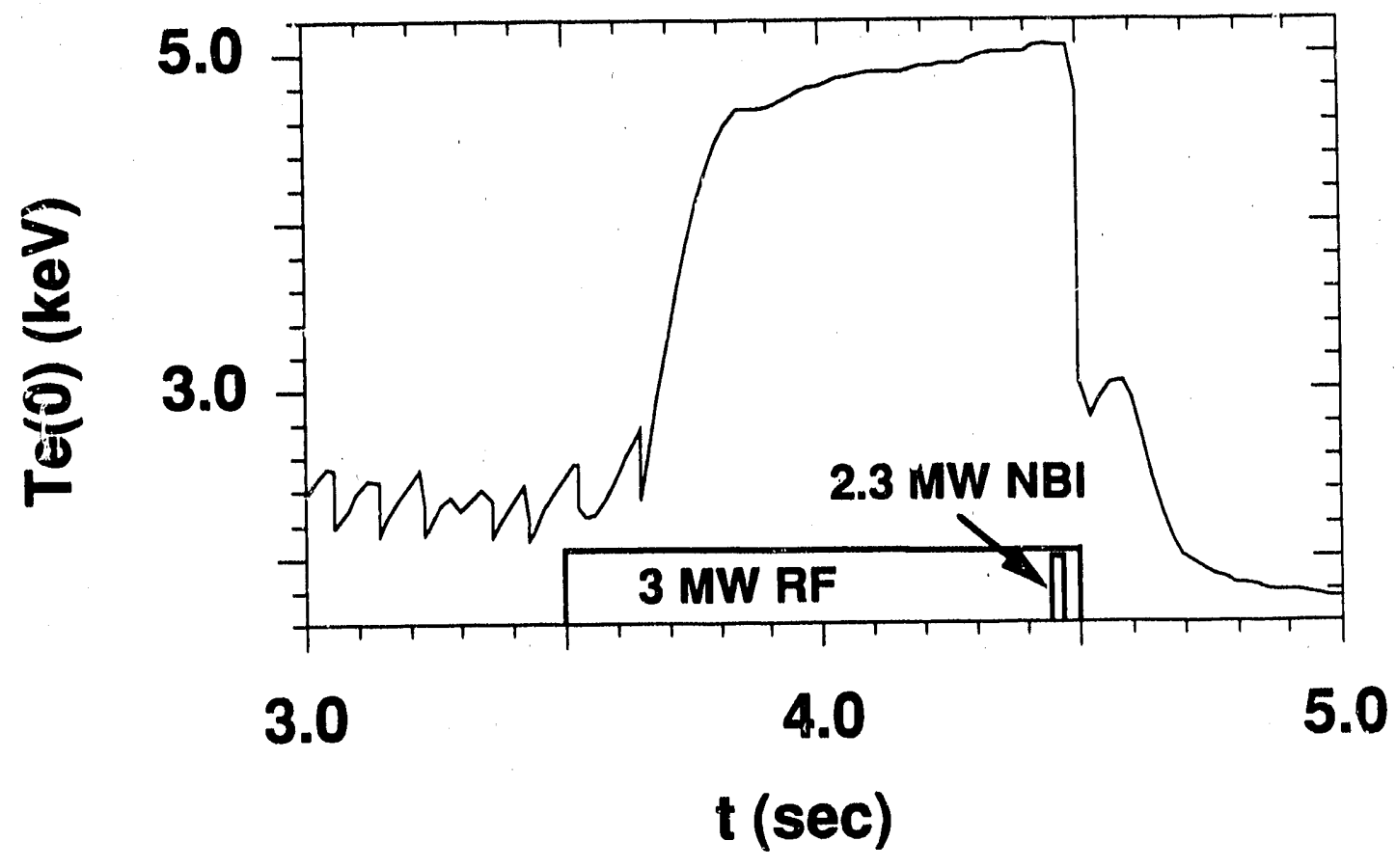

$q(0)$ (calculated) vs. Time

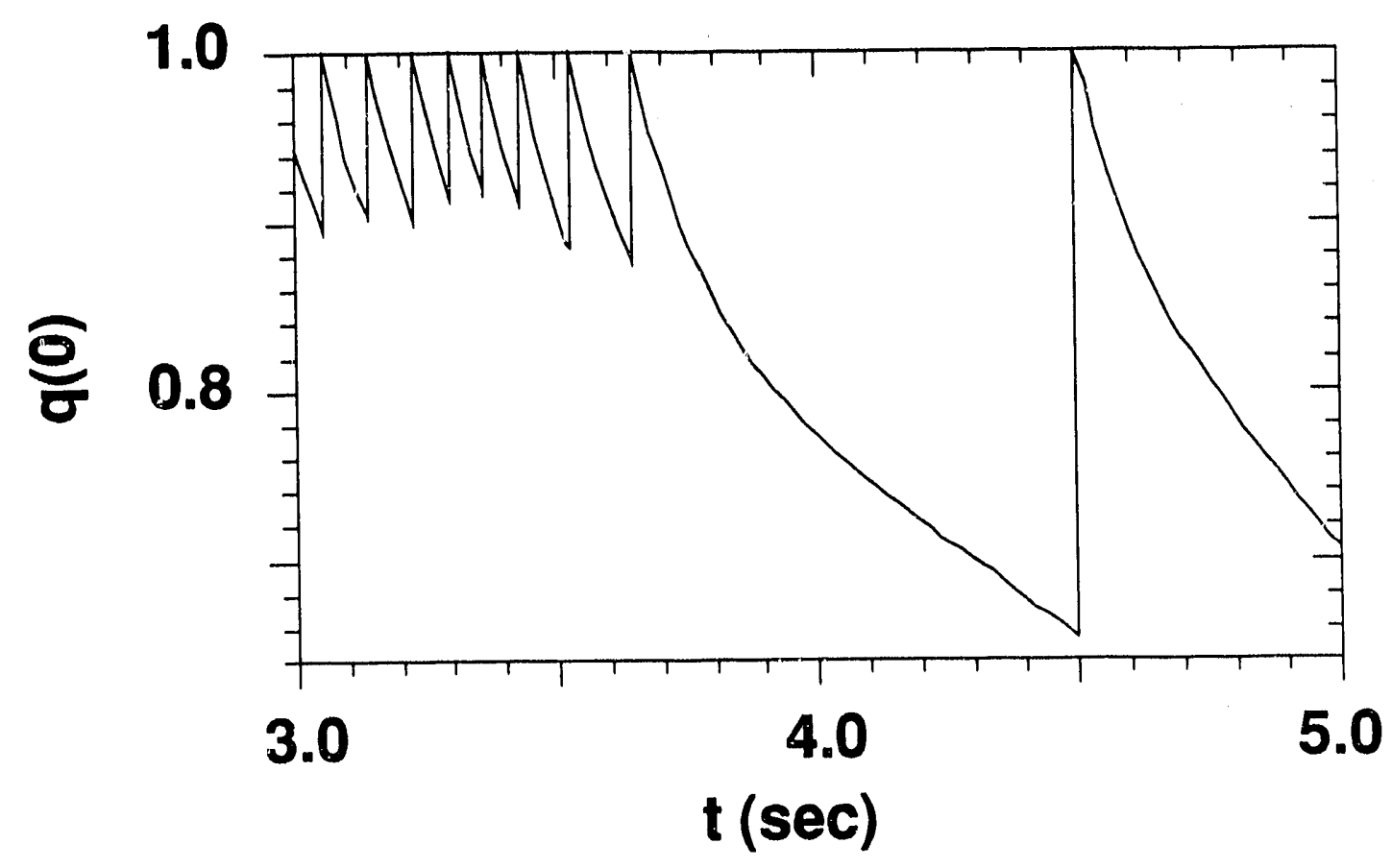

Fig. 9 


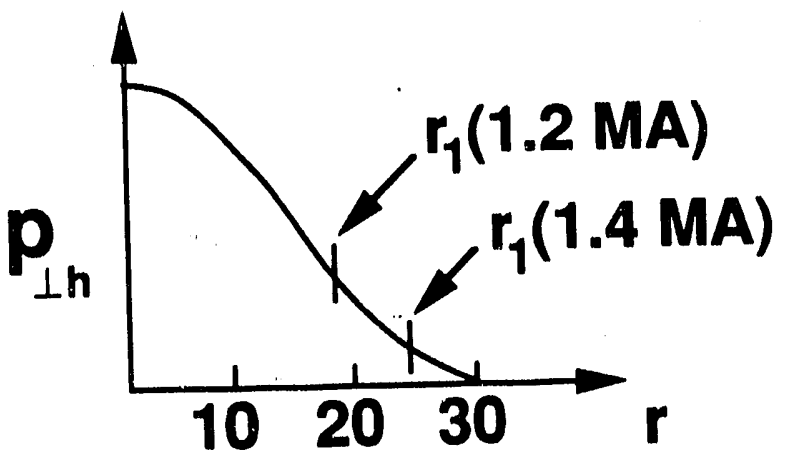

Fig. 10 


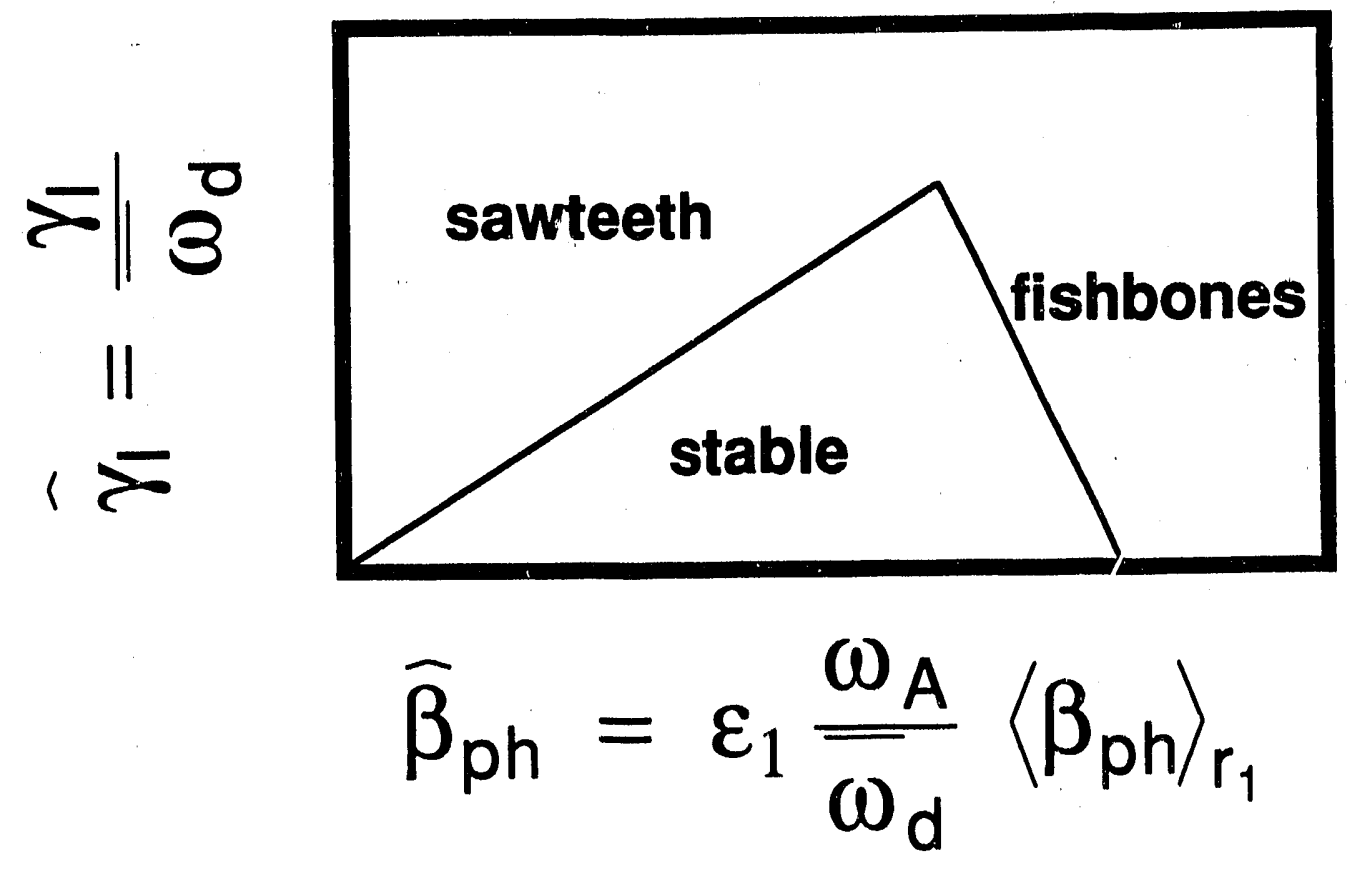

Fig. 11 


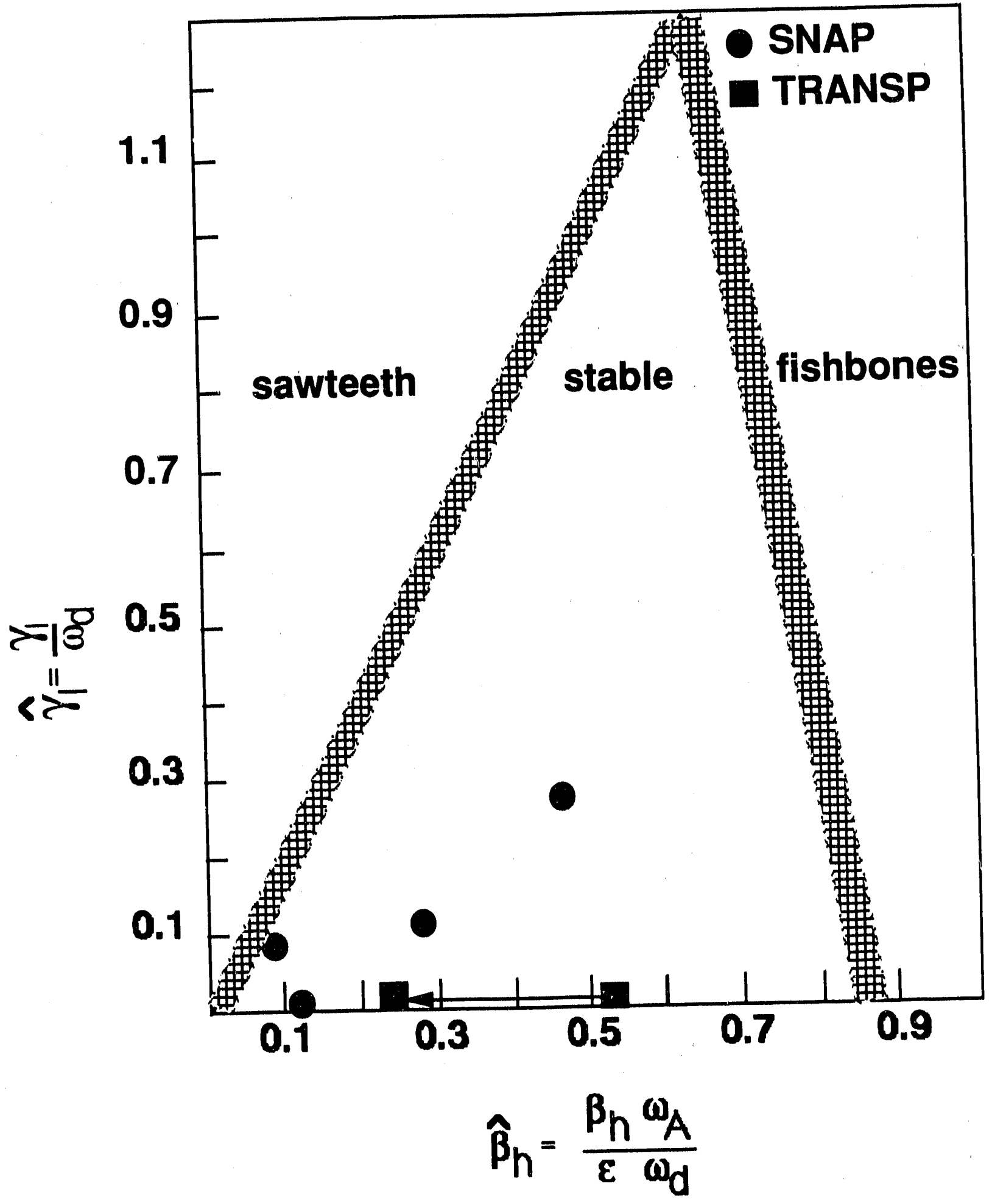

Fig. 12 


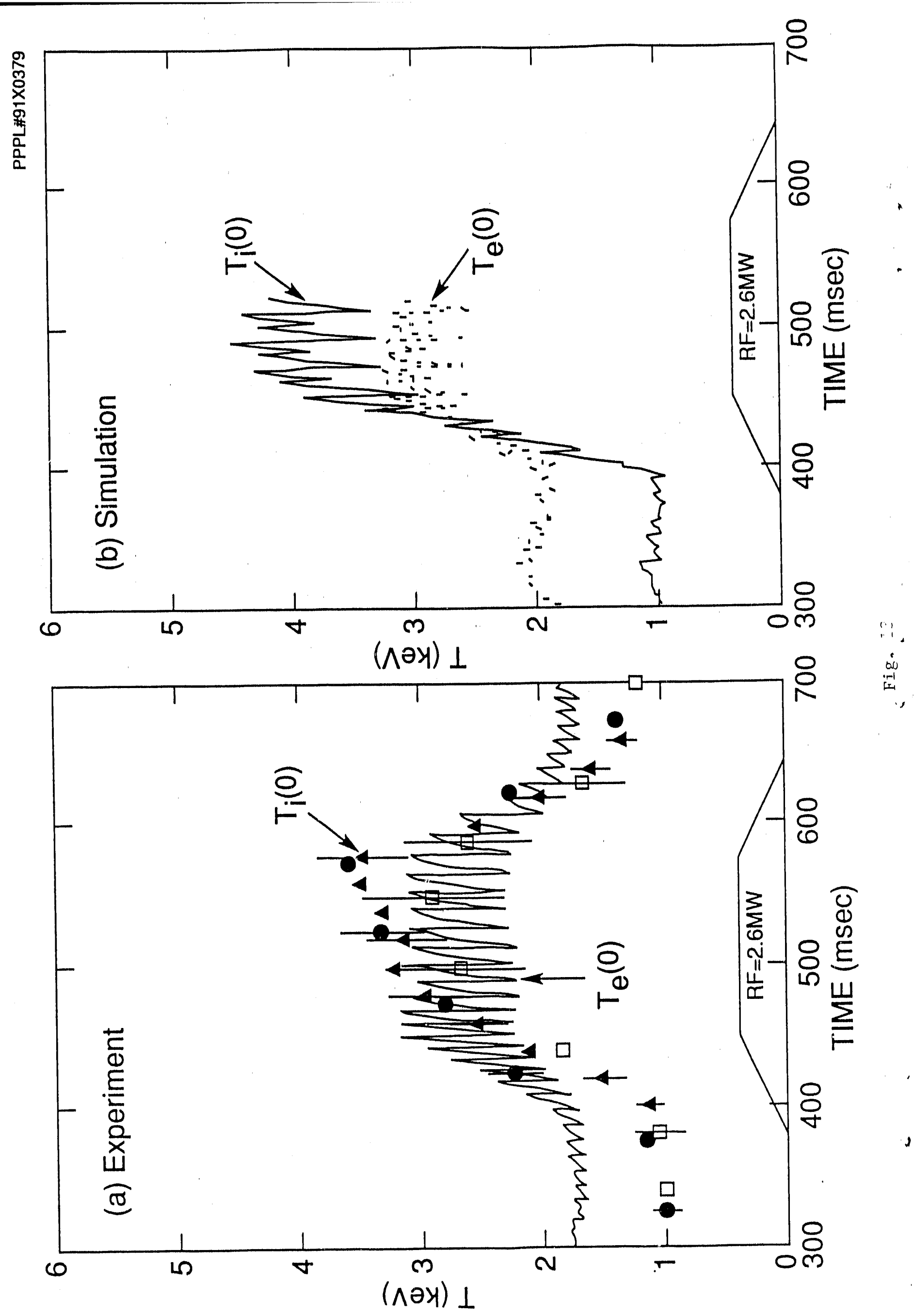


PPPL\#91X0381

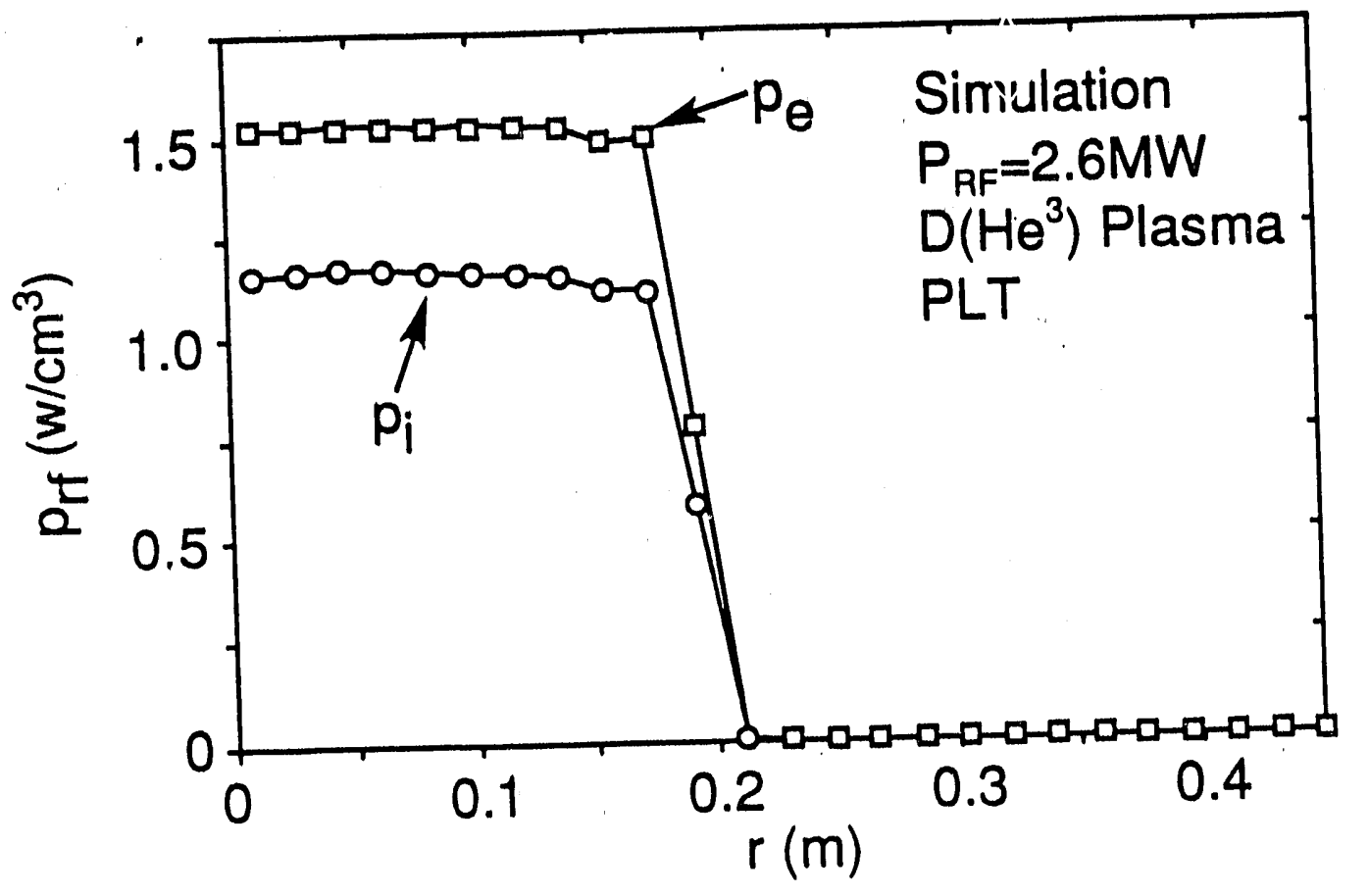

Fig. 14 
PPPL\#91X0230

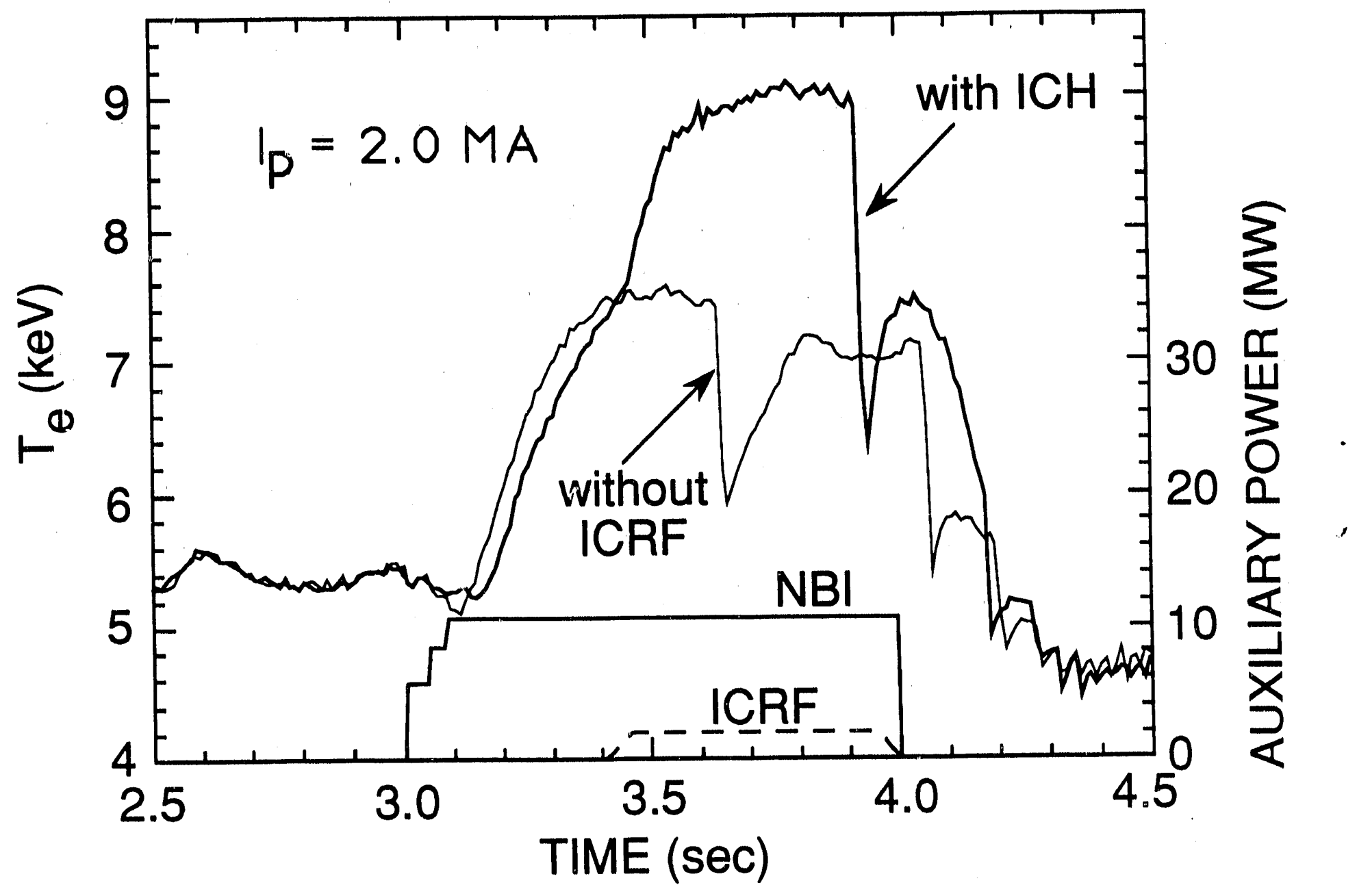

Fig. 15 


\section{EXTERNAL DISTRIBUTION IN ADDITION TO UC-420}

Dr. F. Paoloni, Univ. of Wollongong, AUSTRALIA

Prof. M.H. Brennan, Univ, of Sydney, AUSTRALIA

Plasma Research Lab., Australlan Nat. Univ., AUSTRALIA

Prof. I.A. Jones, Flinders Univ, AUSTRALIA

Prof. F. Cap, Inst. for Theoretical Physics, AUSTRIA

Prof. M. Heinder, Institut fur Theoretische Physik, AUSTRIA

Prof. M. Goossens, Astronomisch Instituut, BELGIUM

Ecole Royalo Milltaire, Lab. de Phy. Plasmas, BELGIUM

Commission-Europeen, DG. XII-Fusion Prog., BELGIUM

Prot. R. Boucique, Rilksuniversiteit Gent, BELGIUM

Dr. P.H. Sakanaka, Instituto Fisica, BPAZIL

Instiluto Nacional Do Pesquisas Espadiais-INPE, BRAZIL

Documents Office, Atomic Energy of Canada Lid., CANADA

Dr. M.P. Bachynski, MPB Technologies, Inc., CANADA

Dr. H.M. Skarsgard, Univ. of Saskatchowan, CANADA

Prof. J. Telchmann, Univ. of Montreal, CANADA

Prof. S.R. Sroenivasan, Univ. of Calgary, CANADA

Prof. T.W. Johnston, INRS-Energie, CANADA

Dr. R. Bolton, Centre canadien de fusion magnétique, CANADA

Dr. C.R. James, Univ, of Alberta, CANADA

Dr. P. Lukác, Komenského Universzita, CZECHO-SLOVAKIA

The Librarian, Culham Laboratory, ENGLAND

Library, R61, Ruthertord Appleton Laboratory, ENGLAND

Mrs. S.A. Hutchinson, JET Library, ENGLAND

Dr. S.C. Sharma, Univ. of South Pacific, FIJI ISLANDS

P. Mahoonen, Univ. of Helsinki, FINLAND

Prot. M.N. Bussac, Ecole Polytechnique, FRANCE

C. Mouttet, Lab. de Physique des Milloux lonisés, FRANCE

J. Radet, CEN/CADARACHE - Bat 506, FRANCE

Prof. E. Economou, Univ. of Crete, GREECE

Ms. C. Rinni, Univ. of loannina, GREECE

Dr. T. Mual, Academy Bibliographic Ser., HONG KONG

Preprint Library, Hungarian Academy of Sci., HUNGAPY

Dr. B. DasGupta, Saha inst. of Nucloar Physics, INDIA

Dr. P. Kaw, Inst. for Plasma Research, INDIA

Dr. P. Rosenau, Israel Inst. of Technology, ISRAEL

Librarian, International Center for Theo Physics, ITALY

Miss C. De Palo, Associazione EURATOM-ENEA, ITALY

Dr. G. Grosso, Istituto di Fisica del Plasma, ITALY

Prof. G. Rostangni, istituto Gas lonizzati Dol Cnr, ITALY

Dr. H. Yamato, Toshiba Res a Devel Center, JAPAN
Prof. I. Kawakami, Hiroshima Univ., JAPAN

Prot. K. Nishikawa, Hiroshima Univ., JAPAN

Director, Japan Atomic Energy Research Inst., JAPAN

Prot. S. $100 h$, Kyushu Univ., WIAPAN

Research Into. Ctr., National Instit. for Fusion Science, JAPAN

Prof. S. Tanaka, Kyoto Univ., JAPAN

Library, Kyolo Univ., JAPAN

Prof. N. Inove, Univ. of Tokyo, JAPAN

Socretary, Plasma Section, Electrotechnical Lab., JAPAN

S. Mori, Tectnical Advisor, JAERI, JAPAN

Dr. O. Mitaral, Kumamoto Inst. of Technology, JAPAN

J. Hyeon-Sook, Korea Atomic Energy Research Inst., KOREA

D.I. Chod, The korea Adv. Inst. of Sa. \& Toch., KOREA

Prot. B.S. Liley, Univ. of Waikato, NEW ZEALAND

Inst of Physics, Chinese Acad Sci PEOPLE'S REP. OF CHINA

Library, Inst. of Plasma Physics, PEOPLE'S REP. OF CHINA

Tsinghua Univ. Library, PEOPLE'S REPUBLIC OF CHINA

Z. Li, S:W. Inst Physics, PEOPLE'S REPUBLIC OF CHINA

Prot. J.A.C. Cabral, Instituto Superior Tecrico, PORTUGAL

Dr. O. Petrus, ALI CUZA Univ., ROMANIA

Dr. J. de Villiers, Fusion Studies, AEC, S. AFRICA

Prof. M.A. Hellberg, Univ. of Natal, S. AFRICA

Prof. D.E. Kim, Pohang Inst. of Sci. Toch., SO. KOREA

Prof. C.I.E.M.A.T, Fusion Division Library, SPAIN

Dr. L. Stenflo, Univ. of UMEA, SWEDEN

Lubrary, Royal Inst. of Technology, SWEDEN

Prof. H. Wilhelmson, Chalmers Univ. of Tech., SWEDEN

Centro Phys. Des Plasmas, Ecole Polytech, SWITZERLAND

Bibliotheok, Inst. Voor Plasma-Fysica, THE NETHERLANDS

Asst. Prof. Dr. S. Cakir, Middte East Toch. Uni',., TURKEY

Dr. V.A. Glukhikh, Sci. Res. Inst. Electrophys.I Apparatus, USSR

Dr. D.D. Ryutov, Siberian Branch of Academy of Sa., USSA

Dr. G.A. Eliseov, I.V. Kurchatov Inst., USSR

Libravian, The Ukr.SSR Academy of Sciences, USSR

Dr. L.M. Kovrizhnykh, Inst. of General Physics, USSR

Kemforschungsanlage GmbH, Zentralbibliothok, W. GERMANY

Bibliothek, Inst. Für Plasmatorschung, W. GERMANY

Prof. K. Schindler, Ruhr-Universitát Bochum, W. GERMANY

Dr. F. Wagner, (ASDEX), Max-Planck-Institut, W. GERMANY

Librarian, Max-Planck-Institut, W. GERMANY

Prot. R.K. Janev, Inst. of Physics, YUGOSLAVIA 

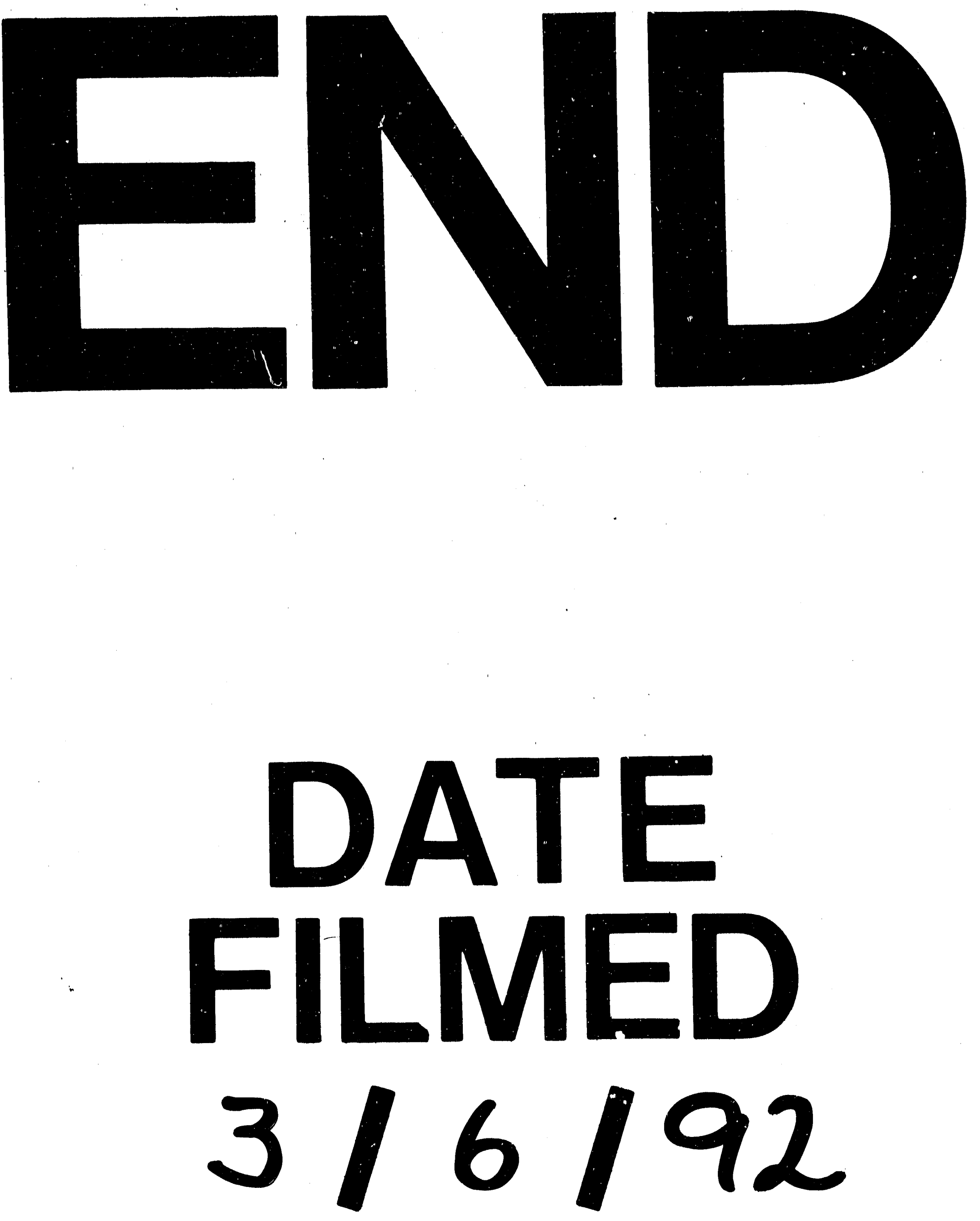